\title{
Carbon nanotubes and graphene towards soft electronics
}

\author{
Sang Hoon Chae $e^{1,2}$ and Young Hee Lee ${ }^{1,2^{*}}$
}

\begin{abstract}
Although silicon technology has been the main driving force for miniaturizing device dimensions to improve cost and performance, the current application of $\mathrm{Si}$ to soft electronics (flexible and stretchable electronics) is limited due to material rigidity. As a result, various prospective materials have been proposed to overcome the rigidity of conventional Si technology. In particular, nano-carbon materials such as carbon nanotubes (CNTs) and graphene are promising due to outstanding elastic properties as well as an excellent combination of electronic, optoelectronic, and thermal properties compared to conventional rigid silicon. The uniqueness of these nano-carbon materials has opened new possibilities for soft electronics, which is another technological trend in the market. This review covers the recent progress of soft electronics research based on CNTs and graphene. We discuss the strategies for soft electronics with nano-carbon materials and their preparation methods (growth and transfer techniques) to devices as well as the electrical characteristics of transparent conducting films (transparency and sheet resistance) and device performances in field effect transistor (FET) (structure, carrier type, on/off ratio, and mobility). In addition to discussing state of the art performance metrics, we also attempt to clarify trade-off issues and methods to control the trade-off on/off versus mobility). We further demonstrate accomplishments of the CNT network in flexible integrated circuits on plastic substrates that have attractive characteristics. A future research direction is also proposed to overcome current technological obstacles necessary to realize commercially feasible soft electronics.
\end{abstract}

Keywords: Carbon nanotube; Graphene; Nano-carbon; Soft electronics; Flexible; Stretchable; Transparent conducting film; Thin film transistor

\section{Introduction}

Since the invention of the transistor, the semiconductor industry has affected nearly every aspect of our daily life $[1,2]$. One main stream technological trend of the silicon industry is scaling down the device sizes. For instance, the gate length has been reduced down to $\sim 20 \mathrm{~nm}$ under current optical lithography technique, and the count of transistors in a commercially available CPU numbers more than 5 billion [3]. In spite of the tremendous progress of miniaturized silicon technology, further development to soft electronics is still limited by the rigidity of the materials themselves. Electronic devices on flexible and stretchable substrates, defined as soft electronics, are contrasted to traditional rigid chips using conventional silicon and metals. The strategies for developing soft electronics are driven by

\footnotetext{
* Correspondence: leeyoung@skku.edu

'Center for Integrated Nanostructure Physics (CINAP), Institute for Basic Science (IBS), Suwon 440-746, Republic of Korea

${ }^{2}$ Department of Energy Science, Department of Physics, Sungkyunkwan University (SKKU), Suwon 440-746, Republic of Korea
}

the investigation of new materials which are bendable, twistable, flexible and stretchable. Toward the basic requirement of replacing traditional rigid silicon electronics by new materials, structure engineering, such as structures in "wavy" layouts and the open mesh geometry have also been investigated to achieve stretchability [4-6].

Figure 1 shows the development of materials for achieving soft electronics from traditional rigid chips. Amorphous silicon (a-Si), low temperature polycrystalline silicon (p-Si), semiconducting metal oxides, nanowires, and organic semiconductors are promising candidates for flexible electronics from a materials perspective, but several challenges must be overcome prior to their practical use. a-Si is low-cost and is applicable for large-area displays, but suffers from poor mobility and flexibility [7]. Low temperature $\mathrm{p}$-Si has the advantage of relatively high mobility but has low uniformity and processability [8]. Metal oxides are costly due to the shortage of rare earth elements and display poor environmental stability. Polymers have

\section{苗}




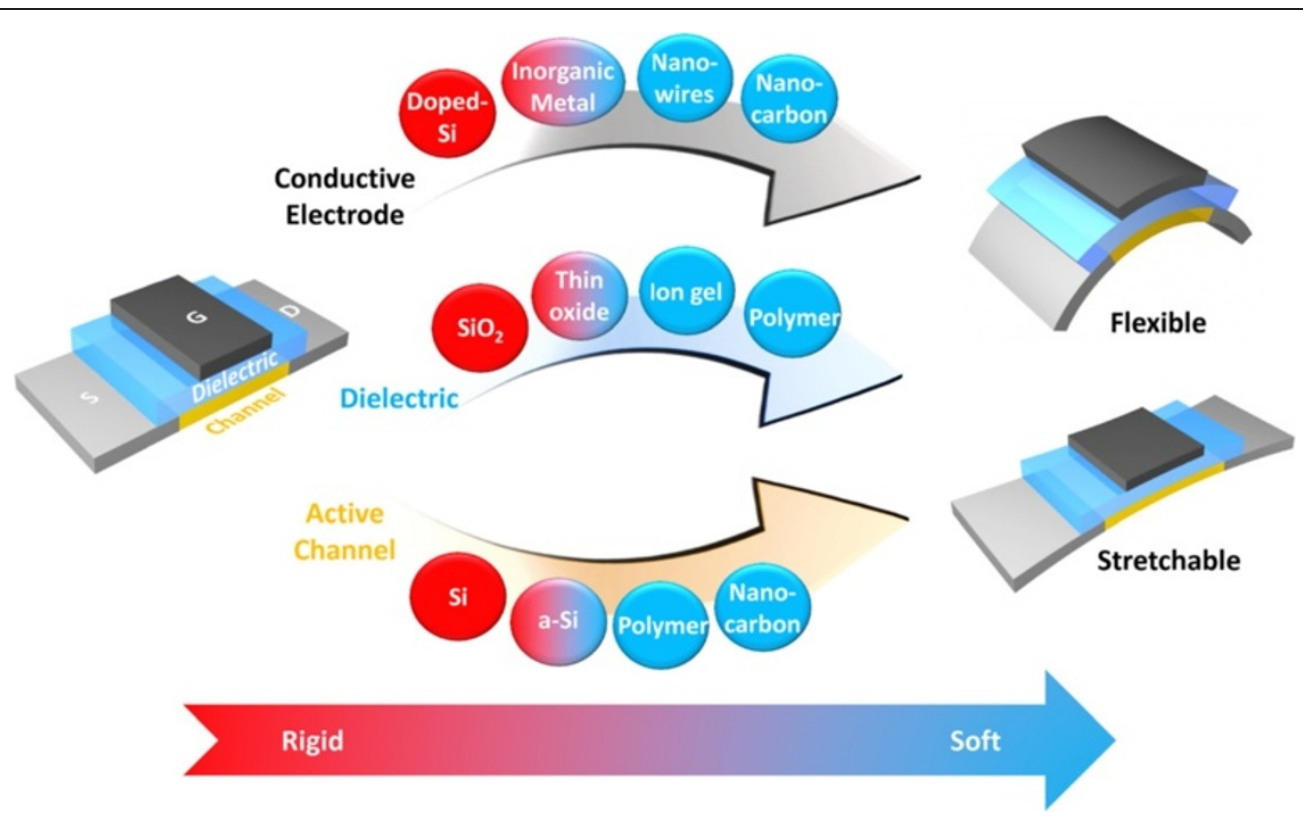

Figure 1 Classification of materials from rigid to soft. Conventional Si-based materials need to be replaced by new materials to realize soft (flexible/stretchable) electronics. With good electrical and mechanical properties, materials such as a-Si, organic polymer, nanowires, and nano-carbon materials are good candidates for next-generation soft applications.

substantial bendability, but have poor mobility and chemical stability.

Nano-carbons such as one-dimensional carbon nanotubes (CNTs) and two-dimensional graphene layers have been widely studied to open a new technology platform based on flexible electronics requiring high transmittance, bendability, and high mobility [9-12]. Figure 2 shows various types of carbon-based materials - fullerene, $\mathrm{CNT}$, graphene, graphite, graphene oxide (GO), and diamond.

The extraordinary electrical, physical, and chemical properties of CNTs and graphene have been attractive since their discoveries. Both materials exhibit outstanding carrier mobility, which is attractive for applications to

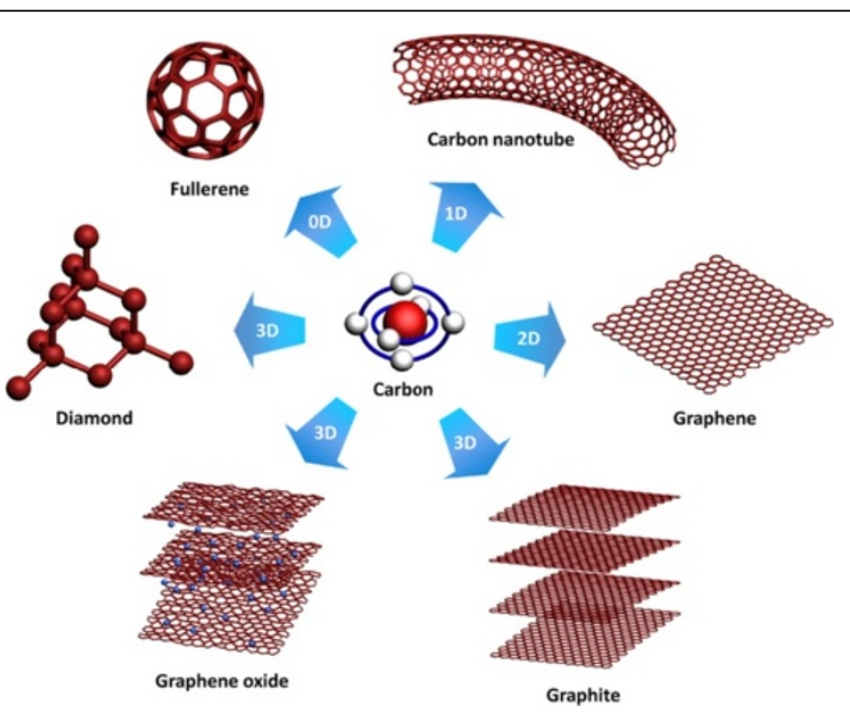

Figure 2 Carbon-based nanomaterials. Nano-carbon materials including OD fullerene, 1D CNT, 2D graphene, 3D graphite, 3D graphene oxide, and $3 \mathrm{D}$ diamond are demonstrated. 
electronic devices. The carrier mobility in semiconducting single-walled carbon nanotubes (SWCNTs) has been shown to be as high as $\sim 80,000 \mathrm{~cm}^{2} \mathrm{~V}^{-1} \mathrm{~s}^{-1}$ [13], while the mobility of exfoliated graphene ranges from $\sim 100,000 \mathrm{~cm}^{2} \mathrm{~V}^{-1} \mathrm{~s}^{-1}$ [14] on insulating substrates to $230,000 \mathrm{~cm}^{2} \mathrm{~V}^{-1} \mathrm{~s}^{-1}$ in suspended structures [15]. These ultra-high mobility values suggest that these materials have the potential to outperform established materials for next-generation high-speed electronics. The electric current capacity for both CNTs and graphene are reported above $10^{9} \mathrm{~A} \mathrm{~cm}^{-2}[16,17]$. At room temperature, CNTs exhibit a thermal conductivity up to $3,500 \mathrm{~W} \mathrm{~m}^{-1} \mathrm{~K}^{-1}$ [18], and graphene has a value of $5,300 \mathrm{~W} \mathrm{~m}^{-1} \mathrm{~K}^{-1}$ [19] with a high transmittance of nearly $97 \%$ [20]. In addition to high flexibility and stretchability, both materials also have superb mechanical strength (Young's modulus of 1.0 $\mathrm{TPa}$ and tensile strength of $130 \mathrm{GPa}$ ) [21]. For these reasons, CNTs and graphene are regarded as the most promising materials to realize next-generation electronics.

The purpose of this article is to summarize the recent progresses of both CNTs and graphene in soft electronics, and furthermore, to provide guidance for future nano-carbon research by clarifying feasible approaches which will most likely lead to soft applications. We first discuss several successful attempts to synthesize CNTs and graphene. Variations in transfer techniques for both materials are discussed thoroughly. For the use of CNTs and graphene for transparent conducting films (TCFs), the characteristics of TCFs using both nano-carbon materials are compared in depth, together with ITO. Furthermore, various types of field-effect devices using different forms of CNT FETs such as single CNT FET, random network CNT FET, aligned CNT FET, and different forms of graphene FETs such as single layer graphene (SLG), bilayer graphene (BLG), and graphene nanoribbon (GNR) are compared. Moreover, the specific FET device performances related to material preparation and fabrication techniques are also discussed. Finally, the logic level, flexibility, and stretchability of devices with a combination of graphene and CNTs along with their utilizations in logic circuits are further discussed. The systematic deep analyses of the device properties of graphene and CNTs highlight excellent opportunities for future flexible electronics. We conclude with a brief perspective on the research directions of soft electronics in future.

\section{Review}

\subsection{Material preparations}

The preparation techniques for CNTs and graphene are the most important fundamental research areas providing realistic applications. From the discovery of CNTs and graphene, diverse work has been done to improve the quality of the materials (crystallinity and uniformity) and to control other parameters (chirality, density, and doping levels) and morphology (length, area, dimension, and thickness). This section describes some of the most successful methods for synthesis of nano-carbon materials.

\subsubsection{Carbon nanotubes}

The CNT synthesis techniques aim to provide control over the tube density, spatial distribution, length, and orientation. Controlling the tube diameter and ratio of semiconducting to metallic SWCNTs have been a critical issue for electrical applications [22,23]. The conventional growth methods for large-scale CNTs include arc discharge, laser ablation, and chemical vapor deposition (CVD) [24-28]. While CNTs grown by arc discharge and laser ablation usually have fewer structural defects than those produced by CVD techniques, the CVD method is intrinsically scalable for realistic applications due to its low setup cost, high production yield, and ease of scale-up. Moreover, long average tube lengths can be obtained from CVD method, which lead to generally better electrical properties in CNTs. The challenge to control alignment and geometry of SWCNTs is mitigated by the CVD method as well. As a one-dimensional material, the as-grown CNTs have various geometries, as shown in Figure 3.

Individual CNTs are horizontally grown on the substrate by CVD, as shown in Figure 3a. Horizontally aligned SWCNTs can be grown using stable and laminar gas flow, which can be determined by the Reynolds number, which depends on volumetric flow rate, viscosity of gases, and the hydraulic diameter of the quartz tube $[29,30]$. Both the buoyancy effect induced by gas temperature and gas flow stability play a dominant role in preparing batch-scale SWCNT arrays [31]. In Figure 3b shows scanning electron microscopy (SEM) images of an aligned SWCNT film grown from Fe catalyst patterned into narrow stripes oriented perpendicular to the growth direction on quartz [32]. The CVD process on ST-cut quartz wafers using patterned stripes of Fe catalyst leads to the highest levels of alignment and density of CNTs. Linear alignment of individual SWCNTs was achieved with an average diameter of $\sim 1 \mathrm{~nm}$, and a density approaching $\sim 10 \mathrm{SWCNT} / \mu \mathrm{m}$. Figure $3 \mathrm{c}$ shows that vertically stacked CNT films can stand on a $\mathrm{SiO}_{2}$ substrate. The CVD growth was carried out on various catalysts, including Fe nano-particles and metal thin films (Fe, $\mathrm{Al} / \mathrm{Fe}, \mathrm{Al}_{2} \mathrm{O}_{3} / \mathrm{Co}$ ) on $\mathrm{Si}$ wafers, quartz, and metal foils to synthesize CNT forest [33,34]. Depending on the collection time, the thickness of CNT films can be changed from micrometers to a few centimeters [35]. Highly-stacked nanotube structures were successfully fabricated on wafer-scale substrates with different thicknesses, which are robust for numerous applications as a conducting film [36,37]. Efficient field emission has been demonstrated where the screening of the field emission current is determined by the ratio of 


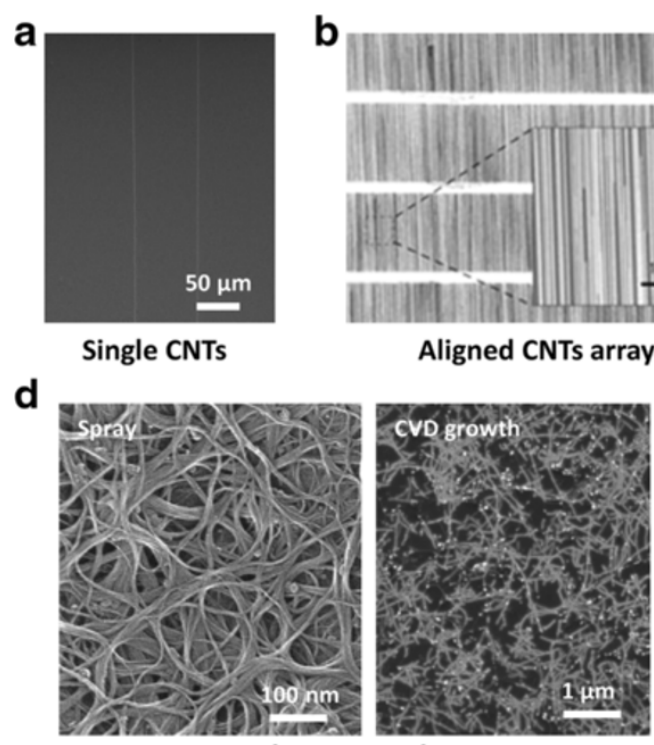

Random network CNTs

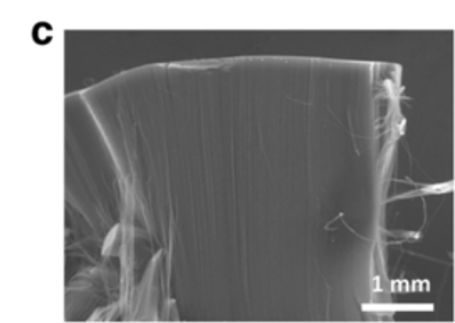

Vertically aligned CNTs

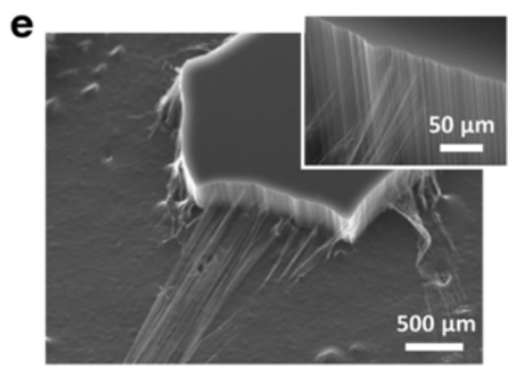

CNT yarning

Figure 3 Various methods of CNT film preparation. a, CVD-grown aligned individual SWCNTs on $\mathrm{SiO}_{2}$ substrate using stable and laminar gas flow. b. Aligned array of CNTs on ST-cut quartz with narrow strip pattern of Fe catalyst. Reproduced with permission [32]. Copyright 2007, Nature Publishing Group. c, Array of vertically aligned MWCNTs on Fe/Al/SiO 2 substrate. d, Random network SWCNTs prepared by spray of CNT solution (left) and CVD-grown on $\mathrm{SiO}_{2}$ substrate (right). e, Yarning of vertically aligned MWCNT film.

the interlayer spacing to CNT length [38,39]. Figure 3d shows SEM images of random network geometry CNTs. The network geometry can be achieved easily by printing SWCNTs from a solution suspension [40,41]. Solution methods such as spray, filtering, dip-coating, and ink-jet printing have been commonly used for random network type CNT films [42-46]. One serious drawback of the solution approach is the bundling of individual CNTs. This degrades the performance of transparent conducting films (sheet resistance vs transmittance) and transistors (on/off ratio vs mobility) [47]. Random network CNT films prepared directly from CVD or arc discharge can also produce CNT networks and improve the device performance $[48,49]$. The bundling of CNTs can be avoided and rather clean CNTs can be retained through the CVD method without worrying about the addition of additives that are used in solution approach [41]. By controlling the concentration of catalysts of $\mathrm{Fe} / \mathrm{Co} / \mathrm{Mo}$, the density of CNTs can be modified, due to increased surface area, pore volume, and catalytic activity [50]. Nevertheless, realizing large-area with good uniformity is still challenging with the CVD method. Owing to their strength, toughness, capabilities of mechanical energy damping, and resistance to knotinduced failure, yarns made from vertically aligned films of MWCNTs are promising multifunctional materials [51-53]. Figure 3e shows an example of the yarning process for a vertically aligned MWCNT film. A beneficial feature of these yarns is the diameter, which can be as little as $2 \%$ of the diameter of a human hair, making them ideal as an artificial muscle actuator or artificial muscle, and for storing energy as part of a fiber supercapacitor or battery. MWCNT fibers could also replace rigid metal wires in electronic textiles, such as in heated blankets, where the rigidity of the metal wires can be uncomfortable. Replacing wires with conducting fibers can also provide radio or microwave absorption, electrostatic discharge protection, other types of textile heating, or for simple wiring applications such as headphones where flexibility is important $[37,54]$.

\subsubsection{Graphene}

Since graphene was first electrically isolated from graphite using a mechanical exfoliation method, many efforts have been studied to synthesize thin graphene films such as the CVD method, reduction of graphene oxide (GO), epitaxial growth on $\mathrm{SiC}$, and chemical molecular assembly method.

As shown in Figure 4a, the mechanical exfoliation technique offers high quality but small flakes of graphene. Tape was used as the micromechanical cleavage layer to detach graphene samples from graphite. The exfoliation method was followed by the identification and selection of monolayers by using an optical microscopy, scanning electron microscopy (SEM), and atomic force microscopy (AFM) [55,56]. However, the practical use of such a graphene for electronics applications is limited by the tiny size of the exfoliated graphene films, despite 


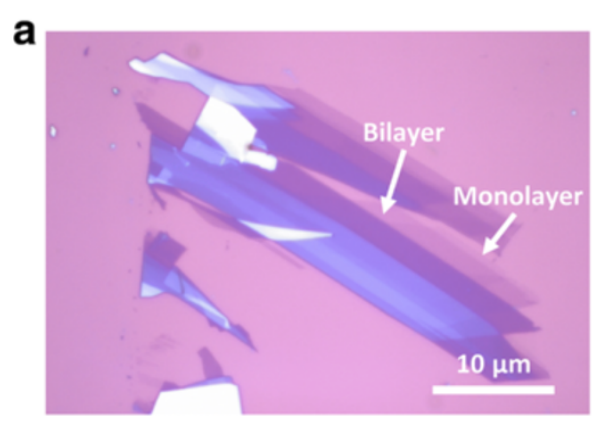

Exfoliated graphene

C
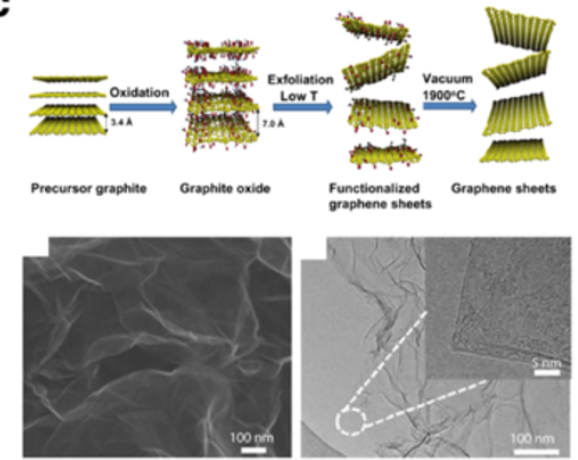

Reduced GO b

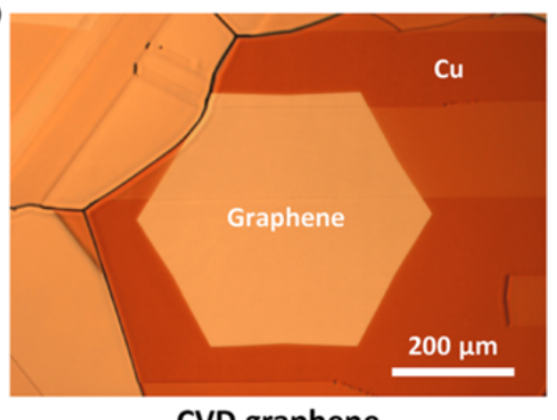

CVD graphene

d
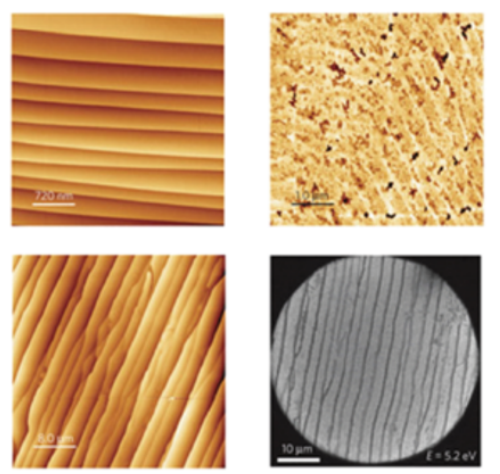

Annealing of SiC

Figure 4 Various methods of graphene synthesis. a, Exfoliated graphene (monolayer, bilayer, and other thick layer) obtained by taping from graphite. $\mathbf{b}$, Graphene flake is grown on Cu foil by CVD. c, Schematic procedure to generate high quality graphene powder obtained from reduced graphite oxide and the electron diffraction pattern. Adapted with permission [73]. d, Images of monolayer graphene on 6H-SiC(0001) for explaining epitaxial growth of graphene. Reproduced with permission [74]. Copyright 2009, Nature Publishing Group.

their high crystallinity. The preparation of graphene using the CVD method has been reported for the feasible use of graphene [57-59]. Figure $4 \mathrm{~b}$ shows that the graphene flake was grown on $\mathrm{Cu}$ foil under an atmospheric CVD system. The CVD approach is attractive because it allows fabrication over large-area, and expansion of the applicability of graphene to flexible or stretchable devices. Although quality and size of graphene keep improving, field effect mobilities of devices using CVD graphene exhibit still lower values compared to those of devices with exfoliated or epitaxial graphene. Yet, the presence of defects such as point defects, grain boundaries, and wrinkles is unavoidable in the CVD process [60]. Grain boundaries and defects reduce the conductivity of the film and therefore it is highly desired to remove them during growth. Observations and controlling such defects are key research topics in the CVD method. Atomic rearrangement at graphene grain boundaries has been observed using transmission electron microscopy (TEM) and scanning tunneling microscopy (STM). Recent works use optical microscopy to observe the grain boundaries realized by selectively oxidizing the underlying copper foil through graphene grain boundaries functionalized with $-\mathrm{O}$ and $-\mathrm{OH}$ radicals generated by ultraviolet irradiation [61] and sodium chloride solution [62]. Graphene can be also prepared by a liquid-phase exfoliation or reduction of GO, which has advantages in quantity, yield and cost [63-67]. Large quantities of GO can be prepared by the traditional Brodie and Hummer method, although these methods can be slightly modified to improve the quality of GO [68-71]. Several reducing agents have been used to achieve reduced GO [72]. Although these methods are advantageous for mass production, the complete removal of epoxy and hydroxyl groups and defect generation are an unsolved problem at the present time, unlike the high quality pristine graphene. A simple thermal exfoliation followed by high temperature annealing up to $1500^{\circ} \mathrm{C}$ in vacuum provides a route of obtaining better quality graphene powder (Figure 4c) [73]. This graphene powder method is challenging but certainly advantageous for conducting film and electrode applications. The fabrication of graphene using the epitaxial growth of graphene directly on rigid insulating silicon carbide $(\mathrm{SiC})$ wafers has been also reported (Figure 4d) [74]. A carbon-included material like $\mathrm{SiC}$ is used as a substrate for graphene preparation with high temperature annealing (around $\left.1,500^{\circ} \mathrm{C}\right)[75]$. Graphene obtained with epitaxial growth 
is highly crystalline, thus is intensely studied to fabricate transistors that operate at high frequencies [76,77]. Wafer-scale graphene can be produced by epitaxial growth on $\mathrm{SiC}$, but those graphenes are not suitable for practical purposes because it is hard to detach graphene from the $\mathrm{SiC}$ substrate. Although a solid source molecular beam epitaxy method was also reported to fabricate graphene directly on $\mathrm{Si}(111)$, the high cost of molecular beam epitaxy will likely prevent the method from being commercially viable [78].

\subsubsection{Transfer methods}

Most CVD approaches for synthesizing CNTs and graphene require high temperatures which prevent direct growth of nano-carbon materials on plastic and other soft target substrates. CNTs and graphene located on a catalytic substrate need to be transferred onto a target substrate. Transferring graphene from the metal substrates onto desired substrates without degrading the quality of graphene is the critical step to use CVD-grown graphene for most practical applications.

Wet etching processes are commonly used to detach as-grown materials from the mother substrates using chemical solutions. $\mathrm{FeCl}_{3}$ or $\left(\mathrm{NH}_{4}\right)_{2} \mathrm{~S}_{2} \mathrm{O}_{8}$ are often used for removing $\mathrm{Cu}$, and $\mathrm{NaOH}$ or $\mathrm{KOH}$ for sapphire $[79,80]$. The most popular binder to hold graphene during wet etching is poly(methyl methacrylate) (PMMA), but this process unavoidably damages and contaminates the graphene layer with residuals, and is not desirable for scale-up fabrication. The dry printing (or stamping) technique uses polydimethylsiloxane (PDMS) stamp to transfer SWCNTs and graphene films from the growth substrates such as $\mathrm{SiO}_{2} / \mathrm{Si}$ and metal films, still has problems with mechanical damage [81]. The roll to roll (R2R) lamination process can produce a large-area graphene film on flexible substrates [82,83]. The R2R transfer technique uses a thermal release layer as a temporary support and enables the continuous production of graphene film on 44 inch-scale flexible substrates. The synthesized graphene with $\mathrm{Cu}$ foil was laminated with the assistance of an adhesive layer, poly(ethylene co-vinyl acetate, EVA) with vinyl acetate (VA) as a supporting layer, to plastic film, followed by $\mathrm{Cu}$ etching, as shown in Figure 5a [82]. The transferred graphene film has appropriate uniformity with a resistance deviation of less than $10 \%$. However, the graphene surface is still contaminated by organic adhesive from the thermal release tape using this transfer approach, which may fairly degrade the electrical properties of the film. Undesired mechanical defects also can be caused by this $\mathrm{R} 2 \mathrm{R}$ transfer on graphene film. A bubbling method for transferring graphene films to target substrates is nondestructive not only to graphene but also to the mothersubstrate (Figure 5b) [84]. The PMMA/graphene/Pt(or $\mathrm{Cu}, \mathrm{Ni}$ ) was dipped into $\mathrm{NaOH}$ solution and was used as the cathode with a constant current supply. At the negatively charged cathode, $\mathrm{H}_{2}$ gas is produced by a water reduction reaction, and the PMMA/graphene layer detaches from Pt substrate due to the $\mathrm{H}_{2}$ bubbles at the interface between the PMMA/graphene and Pt substrate. Damage of the mother-substrate is reduced considerably, and the substrate can be used repeatedly for the next CVD growth. In addition, the transferred graphene is free of metal particles, which are commonly found in graphene transferred by the metal etching process. Figure $5 \mathrm{c}$ explains the "clean-lifting transfer (CLT)" method, which uses electrostatic forces to transfer graphene onto target substrates, and doesn't use a PMMA adhesive layer [85]. An electrostatic generator (SIMCO, $18 \mathrm{kV}$ ) was placed at a distance of one inch away from the substrate, then the discharge process occurred via the electrostatic generator, followed by a pressing process to enable more uniform attachment between graphene and substrate. After the $\mathrm{Cu}$ foil was etched, the remaining graphene film on the target substrate was rinsed with deionized water to remove the residual etchant. The methods described so far are a rather simple transfer process that does not take account of positioning. There is an interesting transfer method for aligning $2 \mathrm{D}$ flakes to a desired location. In order to fabricate stacked graphene on BN devices, a few-micro-size flakes of graphene and BN should be positioned at a desired location (Figure 5d) [86]. Graphene was exfoliated separately onto a polymer stack consisting of a water-soluble polyvinyl alcohol (PVA) and a PMMA layer. When dipped into water, PVA was dissolved and the graphene/PMMA layer was detached from substrate and was floated on the surface of water bath. The PMMA membrane was securely adhered to a holder, which has a tiny hole to identify the top flake onto the PMMA layer during the aligned transfer process. The holder was clamped on the arm of a micro-positioner and then mounted on an optical microscope. The graphene was precisely aligned to the target $\mathrm{BN}$ flake by using the microscope to locate the position and the two (PMMA/ graphene and $\mathrm{BN}$ ) brought into contact. The demand for stacked layered structures has been growing [87-90]. A better strategy for transfer in a large-area without damages and residues on graphene is required for profound study.

\subsection{Carbon-based elements}

Common electronic devices require conducting, semiconducting, and insulating materials. For conducting elements, several conducting polymers such as polyacetylene, polypyrrole, polythiophene, polyaniline, and poly(3,4-ethylenedioxythiophene) poly(styrenesulfonate) (PEDOT:PSS) have been investigated for future applications to replace conventional rigid conducting and semiconducting materials $[91,92]$. However, these polymers have a relatively 
a

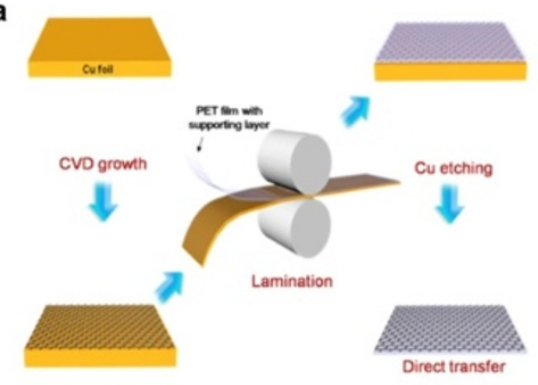

Roll-to-roll lamination transfer

c

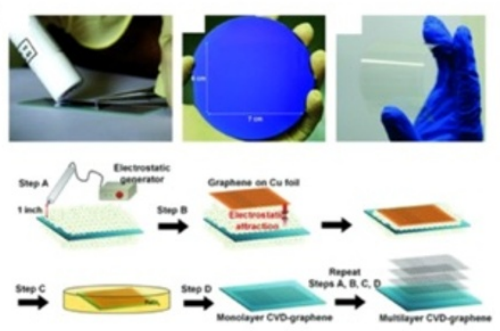

Electrostatic attraction transfer b

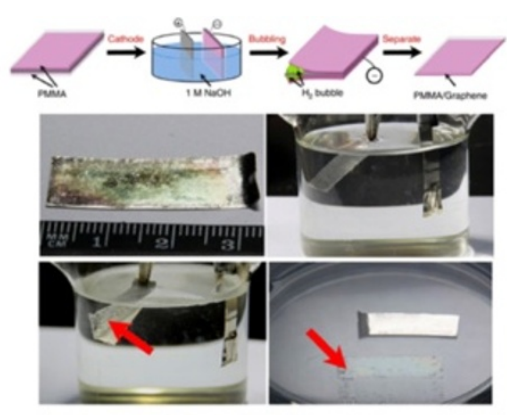

Bubbling transfer

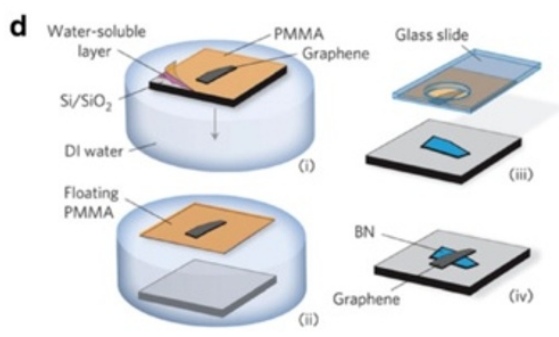

Aligned transfer

Figure $\mathbf{5}$ Various transfer methods of graphene. a, Schematic demonstration of Roll-to-Roll lamination transfer using a thermal release layer. Adapted with permission [82]. b, Schematic and photography images of bubbling process. The PMMA/graphene/Pt was dipped into $\mathrm{NaOH}$ solution with a constant current supply. Reproduced with permission [84]. Copyright 2012, Nature Publishing Group. c, "Clean-lifting transfer $(C L T)^{\prime \prime}$ method, which uses electrostatic forces to transfer graphene. Adapted with permission [85]. $\mathbf{d}$, Aligned transfer for placing graphene and BN to a desired location. Reproduced with permission [86]. Copyright 2010, Nature Publishing Group.

low electrical conductance and poor stability, compared with metal electrodes [93]. a-Si, p-Si, semiconducting metal oxides, nanowires, and organic semiconductors are promising candidates for the active channel, but several challenges - including rigidity and electrical performance issues - must be overcome prior to practical uses. CNTs and graphene electrodes can be an alternative not only to conducting electrodes but also to a semiconducting channel.

\subsubsection{Conducting electrodes}

Electrical conducting materials would have potential for consumer applications, such as soft displays, energy generators, and human bio-devices. In such applications, metal oxides such as IZO and ITO are the most widely used materials [94-96]. However, they have several limitations: i) They are costly and a predicted shortage of indium is a concern, and ii) fracture strain less than $1 \%$ limits the mechanical ability of flexible devices. Nanocarbon materials can overcome many of these limitations and open a new technology platform due to their outstanding electronic, optoelectronic, thermal, and mechanical properties. Here, we describe nano-carbon materials as conductive electrodes and the development of TCF using CNTs and graphene, where the aim is to replace ITO for certain applications.
During the past few years, much effort has been given in synthesizing CNT films as a conducting element [44,97-99]. Such CNT films have many applications including flexible and stretchable transparent loudspeakers [100], electrodes for LEDs, [101] lithium-ion batteries [102], and touch panels [103]. Figure 6a shows a practical touch panel assembled by directly yarning vertically aligned CNTs. Although the idea of utilizing CNT films as conducting materials is simple, controlling density, average tube length, tube diameter and mixture of metallic and semiconducting CNTs is still challenging. Even with optimized growth conditions, one serious drawback is the relatively high sheet resistance compared to that of conventional ITO [104]. Highly flexible, transparent, and conducting SWCNT films are one of the recent emerging technologies [105-107]. The pristine SWCNT TCF have a reported $360 \Omega /$ sq sheet resistance at transmittance of $90 \%$ [43]. This sheet resistance could be dramatically improved by chemical doping treatments. Once such method using nitric acid removes the remaining surfactant from the CNT network and can lower the sheet resistance to a $150 \Omega / \mathrm{sq}$ at transmittance of $90 \%$ [108]. Further doping with $\mathrm{Au}^{3+}$ ions has also been shown to reduce sheet resistance to $110 \Omega / \mathrm{sq}$ at a transmittance of $90 \%[109,110]$. While not surpassing the electrical performance of ITO, these films have 
a

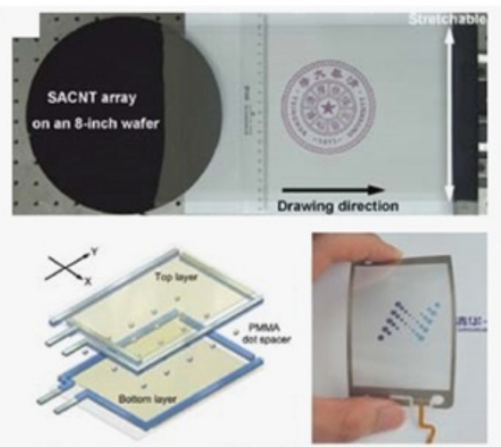

CNT TCF for touch screen

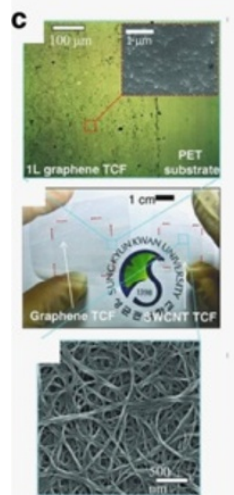

SWCNT vs Graphene TCF

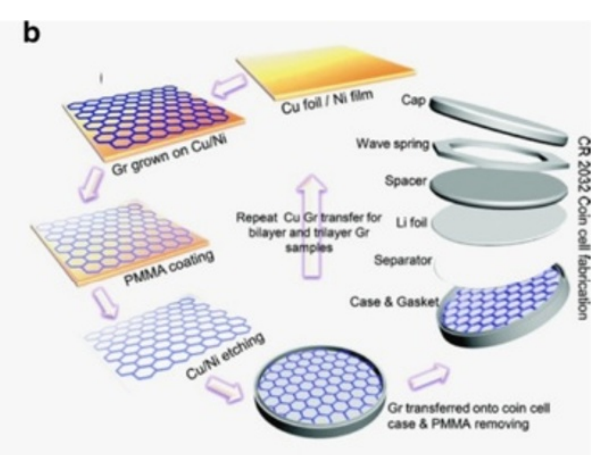

Graphene electrode for battery

d
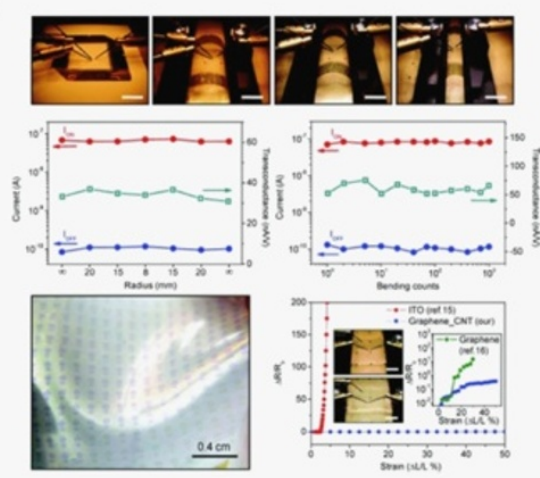

SWCNT/Graphene hybrid film

Figure 6 CNTs and graphene as conducting electrodes. a, Touch screen using yarned CNT film from vertically aligned CNTs. Adapted with permission [103]. b, Li-ion battery using CVD graphene as an electrode. Reproduced with permission [111]. Copyright 2012, American Chemical Society. c, Comparison of the properties (bending angle vs sheet resistance, and transmittance vs sheet resistance) of CNT- and graphene-based TCF with ITO film. Adapted with permission [47]. d, Mechanical advantage of SWCNT/graphene hybrid electrode. Reproduced with permission [123]. Copyright 2011, American Chemical Society.

the advantage of better mechanical stability and are fabricated from a more ubiquitous chemical element, carbon. Figure $6 \mathrm{~b}$ shows an example that graphene can be used as electrodes to study $\mathrm{Li}$ ion diffusion through graphite in lithium-ion batteries [111]. Together with CNTs, graphene is attractive as a conducting film $[112,113]$, due to a large theoretically-predicted conductivity and good chemical stability. In particular, a scalable CVD process to produce large sheets of graphene with high transmittance and robust adhesion to plastic polymers opens the possibility of using graphene in numerous applications in soft electronics. Still the improvement of sheet resistance of the film is an important issue for conducting films. Similar to CNT films, the chemical doping approach has been widely studied for conductivity improvement in graphene films [114-116]. A new approach of layer-by-layer (LbL) doping to improve the conductivity of transparent graphene films has been proposed [117]. Each layer was transferred to a polyethylene terephthalate (PET) substrate followed by $\mathrm{AuCl}_{3}$ doping. This approach demonstrates not only improvement of sheet resistance and uniformity but also better environmental stability compared to topmost layer doping. The optimized LbLdoped four-layer graphene shows a sheet resistance of $54 \Omega / \mathrm{sq}$ and a transmittance of $85 \%$ (at $550 \mathrm{~nm}$ ) with a robust bending stability. The performance of the graphene conducting films need to be further tuned and improved to meet different requirements of practical flexible products $[118,119]$. Both CNTs and graphene TCFs have a remarkable spectral response in the UV region, compared to the poor response of ITO films, as shown in Figure 6c [47]. While ITO shows a rapid increase in the sheet resistance due to cracking of the film as the bending angle increases, SWCNTs and graphene films show almost no significant change in the sheet resistance. One drawback of the CNT TCF film is that the performance strongly relies on the dispersion of CNTs in solution. In graphene case, the bottleneck process is the transfer process, which often involves wrinkles and crack formation. Compared to a two-dimensional graphene film, the SWCNT/graphene hybrid electrode is interesting due to its enhanced mechanical properties 
[120-122]. The SWCNT/graphene hybrid electrode showed a $36 \%$ resistance change at a $50 \%$ strain, as shown in Figure 6d [123]. The resistance change is remarkably smaller than found in ITO electrodes (i.e., 2000\% at 5\% strain) and even in a few layers of graphene (i.e., 200\% at $30 \%$ strain). This superb stretching performance results from the use of graphene and SWCNT network. A continuous and robust contact can be formed between the SWCNT network and the graphene electrode even with graphene layer cracks under strain. This one- and two-dimensional material combination could well provide CNTs and graphene as an appropriate soft and transparent electrode. Table 1 summarizes the transmittance and sheet resistance of various films. It seems that doping is very necessary to reduce sheet resistance. It is also noted that the CNT/graphene hybrid may improve the sheet resistance. This will be a future research direction.

\subsubsection{Active channel - CNT and graphene FETs}

Miniaturization is the most important issue not only to increase device integration density but also to improve FET performance for complicated operations. Semiconducting $\mathrm{Si}$ technology has given great contributions to society, but now faces scaling which involves heat and power consumption issues due to the fundamental limitations of Si. Atomic-thick nano-carbon materials might satisfy the scaling issue and give great benefits with combination of electrical/mechanical/optical advantages. As an active channel component, SWCNTs and graphene have been studied for fabricating FETs and $\mathrm{p}-\mathrm{n}$ junctions to demonstrate their potential to outperform established materials for next-generation electronics [125-128]. Here, we discuss extensively the advantages and challenges of such nano-carbon materials for the use of FETs and furthermore their adaptability to silicon technology.

Figure 7 shows that various kinds of FETs using nanocarbon materials-based active channel. Diverse geometries of FETs based on semiconducting SWCNTs have been the subject of intensive research [129-131]. An individual SWCNT FET shows favorable device characteristics such as large on-off ratio $\left(>10^{5}\right)$, at roomtemperature operation [132-134]. With single CNT studies, it has also been demonstrated that the saturated on-current level can be simply determined from the work function difference between the CNT and metal or Schottky barrier height formed at the junction, as shown in Figure 7a [135]. For fabricating this transistor, e-beam lithography is used to pattern the electrodes to desired positions, but has limitations for realistic multi-array transistors. An alternative easy fabrication method without e-beam lithography is required for large-scale integration for practical electronic device applications. Although isolated SWCNTs are not relevant to future applications at their current stage, numerous works show that the aligned arrays of SWCNTs or random networks can serve as an active channel component. Figure $7 \mathrm{~b}$ shows FETs with aligned arrays of SWCNTs. The use of dense aligned arrays of linear SWCNTs was used as an effective semiconducting channel suitable for integration into transistors and other classes of electronic devices [32]. The tubes were parallel to one another to better than 0.1 degree. The average CNT density can be as high as $10 \mathrm{SWCNT} / \mu \mathrm{m}$, and the film provides good device-level performance characteristics with mobility of $\sim 1,000 \mathrm{~cm}^{2} \mathrm{~V}^{-1} \mathrm{~s}^{-1}[136,137]$. Figure 7c shows an array of FETs with random network SWCNTs that were synthesized on a catalyst $(0.01 \mathrm{M}$ of ferrocene) array by using a plasma-enhanced chemical vapor deposition (PECVD) method at low temperature $\left(450^{\circ} \mathrm{C}\right)$ [138]. SWCNTs network was placed between the source and drain electrodes and played a role of active channel path. This random network type morphology has the potential applicability from CNT thin film transistors (TFTs) to large-scale flexible electronics due to its good uniformity and processability over a large-area, which is alternative to conventional organic or other classes of semiconductors for integrated circuitry applications $[126,139]$. However, the gate modulation is degraded due to the inclusion of some metallic CNTs in the channel. Strategies to reduce metallic CNTs in the channel will be discussed in the next Section 3.2.1. Figure $7 d$ shows an example of graphene channel FETs on a flexible plastic substrate [140]. In graphene, the charge carriers in the two-dimensional (2D) channel can change from electrons to holes subject to electrostatic gate with a minimum

Table 1 Performance comparisons for TCFs based on graphene and carbon nanotubes

\begin{tabular}{|c|c|c|c|c|c|}
\hline Material & Preparation method & $\begin{array}{c}\text { Transmittance } \\
\text { (\% at } 300 \Omega / s q \\
\text { sheet resistance) }\end{array}$ & $\begin{array}{c}\text { Sheet resistance } \\
(\Omega / \mathrm{sq} \text { at } 90 \% \\
\text { transmittance })\end{array}$ & Flexibility & Stretchability \\
\hline Random network CNTs [108] & Spray \& $\mathrm{AuCl}_{3}$ doping & 95.7 & 110 & $\mathrm{O}$ & $\mathrm{O}$ \\
\hline Yarning CNTs [103] & Laser trimming \& Metal deposition & 91 & 208 & $\mathrm{O}$ & $\mathrm{O}$ \\
\hline CVD Graphene [117] & Layer-by-layer doping & 97 & 108 & $\mathrm{O}$ & $\mathrm{O}$ \\
\hline CNT-Graphene hybrid [123] & Solid-phase layer-stacking & 70 & 735 & $\mathrm{O}$ & 0 \\
\hline Metal-Graphene hybrid [124] & Metal grid \& Graphene transfer & - & 20 & $\mathrm{O}$ & $\mathrm{O}$ \\
\hline ITO [104] & Sputtering & 91 & 80 & Poor & - \\
\hline
\end{tabular}


a
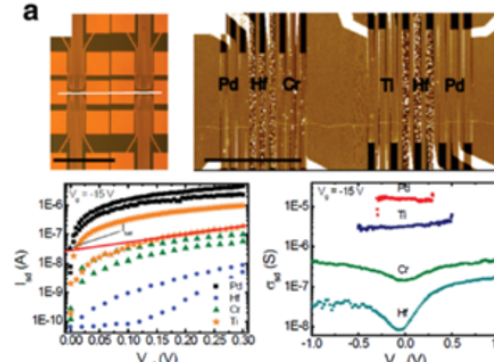

$v_{x i}(n)$

Single CNT transistor

C
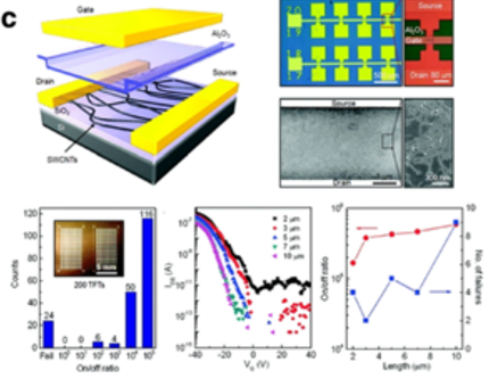

Random network CNT transistor b
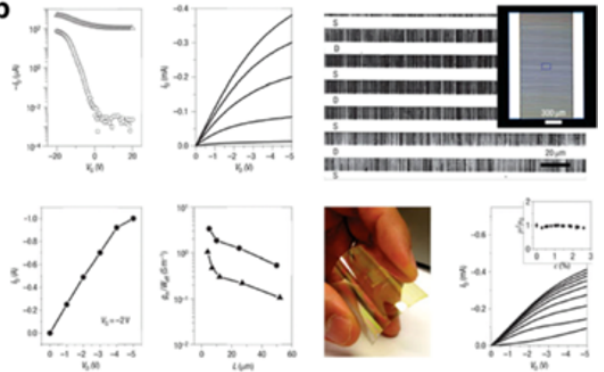

Align CNT transistor

d
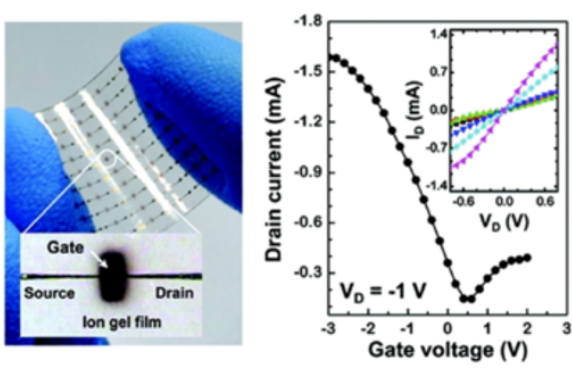

Graphene transistor

Figure 7 Morphologies and characteristics of CNTs and graphene FETs. a, Single CNT transistors with different metal electrodes ( $\mathrm{Pd}$, Hf, $\mathrm{Cr}$, and Ti). Reproduced with permission [135]. Copyright 2011, American Chemical Society. b, Electrical performance, SEM images, and optical microscopy images of flexible TFTs using aligned CNTs array. Reproduced with permission [32]. Copyright 2007, Nature Publishing Group. c, Array of FETs with random network SWCNTs. Reproduced with permission [138]. Copyright 2009, American Chemical Society. d, Flexible graphene transistor with ion gel dielectric. Reproduced with permission [140]. Copyright 2010, American Chemical Society.

density (or Dirac) point characterizing the transition [127,141-143]. The experimental graphene FETs have extremely large mobility compared to SWCNT FETs, while on/off ratio is as low as $\sim 10$ due to zero band gap. Despite low on/off ratio, high transconductances and current saturation are achieved, making graphene devices suited for analogue applications [144].

\subsubsection{Performance control - on/off ratio control} One of the key issues in high-performance TFTs is high on/off ratio for efficient switching behavior. In the case of a CNT channel, the as-grown CNT network usually contains both semiconducting and metallic CNTs [145]. These metallic CNT paths reduce the on/off ratio of the transistor [146]. Since controlling the ratio of semiconducting to metallic CNTs leads to a trade-off between on/off ratio and charge carrier mobility of a transistor, engineering the proper parameter is important in terms of the type of applications. In the case of zero band gap graphene, opening the band gap is a big challenge in the way of achieving a higher on/off ratio in transistors [127]. Here, we introduce several strategies for increasing the on/off ratio of a transistor. In CNTs, electrical thinning and selective channel cutting, and separation approaches are described below. BLG and nanoribbon approaches will be discussed for increasing the on/off ratio in graphene transistors.

One method to obtain high on/off ratio involves electrical thinning of the thick MWCNTs and CNT bundles, as shown in Figure 8a [147]. The electrical thinning process involves sweeping the drain voltage from $0 \mathrm{~V}$ to negative values while holding the gate voltage at a just above the threshold. Multiple sweeps with increasing voltage eventually eliminate metallic CNT channels or thin nanotubes (or bundles) to increase on/off ratio $[32,148]$. After this procedure the off-state current in the devices is reduced to values consistent with semiconducting CNTs alone. A striping technique was used to cut metallic CNT paths $[123,149]$. Figure $8 \mathrm{~b}$ shows the schematic image and SEM image of a region of the random network SWCNT channel. By inserting the cutting line perpendicular to the channel length direction, the metallic CNTs can be terminated and the on/off ratio increases. The critically important role of the cutting width in determining the electrical characteristics can be quantified. For cutting widths of $5 \mathrm{~mm}$, the etched lines increase the on/off ratio by up to four orders of magnitude, while reducing the transconductance by only $40 \%$. It is now possible to obtain uniform CNT thin films with only semiconducting behavior by the techniques of 
a
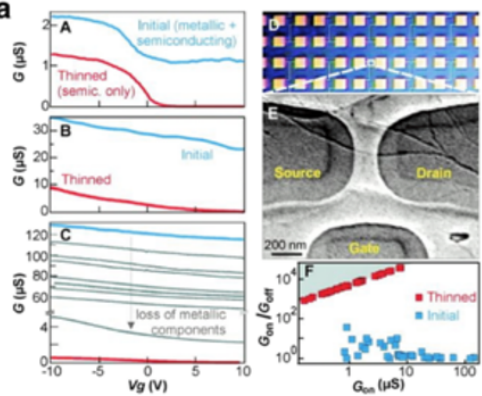

Electrical thinning

d
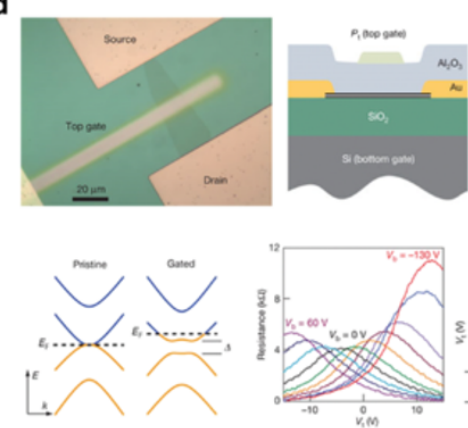

Bilayer graphene b

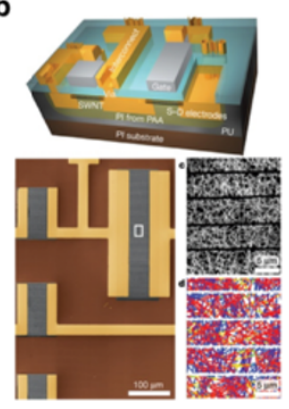

Metallic cutting c
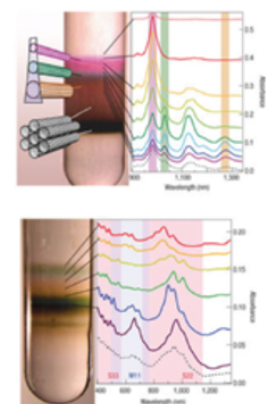

Separation

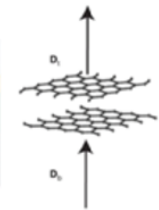

e
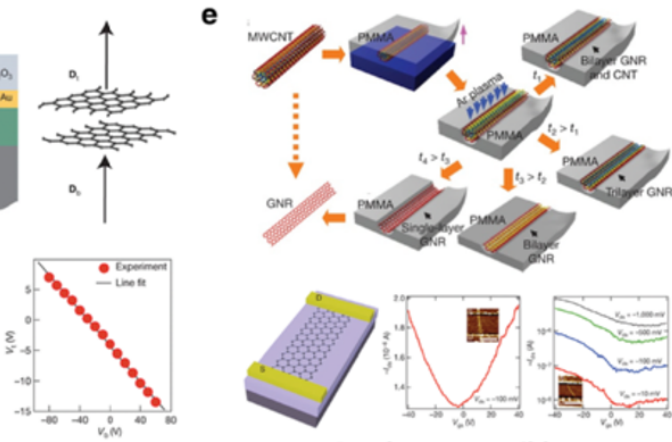

Graphene nanoribbon

Figure 8 Various methods of improving on/off ratio of FET based on CNTs and graphene. a, Thinning of MWCNTS and CNT bundles by applying bias. Reproduced with permission [147]. Copyright 2001, American Association for the Advancement of Science. b, Schematic and SEM image of a region of the random network SWCNT channel. A striping technique was used to cut metallic CNT paths. Reproduced with permission [149]. Copyright 2008, Nature Publishing Group. c, Separation of semiconducting CNTs and metallic CNTs by density-gradient method. Reproduced with permission [153]. Copyright 2006, Nature Publishing Group. d, BLG transistor with top and bottom gate to open band gap. Applying perpendicular field from bottom gate, band gap of the BLG can opened up to $250 \mathrm{meV}$. Reproduced with permission [157]. Copyright 2009, Nature Publishing Group. e, Graphene nanoribbons with a width below $10 \mathrm{~nm}$ were obtained by upzipping CNTs. By narrowing the width of graphene to a few nanometers, a quantum confinement effect of carriers happens to open the band gap. Reproduced with permission [162]. Copyright 2009, Nature Publishing Group.

semiconducting/metallic CNT separation in solution [150-152]. The purification processes produce separated CNTs in solution of the same chirality, diameter, length and semiconducting/metallic type. A self-sorting method to achieve a chirality separated CNT thin film by controlling surface chemistry and a further large-scale demonstration was reported in Figure 8c [153]. The representative techniques are density gradient ultracentrifugation (DGU) and gel chromatography, which can produce $>99 \%$ semiconducting CNTs and continue to improve. Despite the quite low productivity, yield, and high process cost, this DGU technique appears to be the most promising method to prepare semiconducting CNT materials [153]. The gel chromatography separation method, much simpler than DGU method, is based on the strength of the structure-dependent interaction of CNTs with an allyl dextran-based gel [152]. TFTs based on such separated CNTs also provide high on/off ratio. BLG has a unique dispersion relationship whereby application of a strong transverse electric field breaks electron-hole inversion symmetry [154-156]. Experimentally, it has been reported that an optical bandgap of $250 \mathrm{meV}$ is possible. The effective electrical gap is smaller than the reported optical gap, typically due to the presence of disorder and sample imhomogeneities. Even so, large improvements in on/off ratios and the existence of an insulating state at charge neutrality have been observed (Figure 8d) [157]. In these dual-gate BLG transistors, on/off ratios of $\sim 100$ and $\sim 2000$ at room temperature and $20 \mathrm{~K}$ have been reported, respectively [158]. BLG is disadvantageous compared to graphene monolayer since acousticphonon scattering is increased strongly, optical-phonon scattering is reduced, and a parabolic band dispersion near the band edge reduces carrier mobility compared with monolayer graphene [159]. Moreover, the band structure of BLG can be modified, with a larger bandgap possible by applying a combination of strain (along $\mathrm{z}$ axis) and an electrical field. However, this approach is unfeasible with current technology. A new strategy demonstrated that benzyl viologen (BV) as an electron- 
donating group and bis(trifluoromethanesulfonyl)imide (TFSI) as an electron-withdrawing group are conjugated on the top and bottom sides of bilayer graphene to open the band gap $[160,161]$. This compensation doping induces a high local electric field in the bilayer, but has the limitation of weak field-effect due to a large disorder potential. The graphene nanoribbon (GNR) strategy is to ideally introduce a quantum confinement effect of carriers to open the band gap by narrowing the width of graphene to a nanometer scale. In reality, this strategy is limited by fabrication procedures. Instead of confinement-induced gap, this leads to a coulomb blockade effect that is strongly enhanced for dimensions below $20 \mathrm{~nm}$. Graphene nanoribbons with a width below $10 \mathrm{~nm}$ can be obtained by upzipping CNTs (Figure 8e) [162] and by solution-phase stripping from bulk graphite [163]. The GNR transistors exhibited an on-off ratio of $\sim 10^{7}$ at room temperature [162-165]. Similar to the GNR method, graphene with a nanomesh structure can open up a band gap and shows an on/off ratio of $>10^{2}$ in a large sheet of graphene [166,167]. However, these GNR and graphene nanomesh transistors have poor on-state conductivity and cannot be used for high-speed devices unless a new method is found due to reduce scattering at the edges. The band gap of graphene can be modulated by chemical and physical doping processes. Band gaps of boron- and nitrogen-doped graphene transistors showed an on/off ratio of $>100[168,169]$. It also has been reported that by patterned adsorption of atomic $\mathrm{H}$ onto the graphene surface, surface absorption can induce a band gap in graphene of at least $450 \mathrm{meV}$ around the Fermi level [170]. Yet, again the degradation of mobility due to $\mathrm{sp}^{3}$ hybridization with atomic $\mathrm{H}$ makes this approach impractical.

2.2.2.2 Performance Control - Polarity Control Although CNTs and graphene intrinsically have an ambipolar transport property, both show p-type behavior under ambient conditions due to contacts, doping by oxidizing acids, or doping by the adsorption of atmospheric oxygen molecules and/or moisture. It is important to control the carrier type of nano-carbon transistors for applying "complementary metal-oxide-semiconductor (CMOS) technology" because high noise immunity and low static power consumption are critical issues in the modern semiconductor industry. Therefore, it is desired to control the major carrier types of CNTs and graphene FETs by chemical and/or nonchemical doping methods. Here, we introduce several polarity control methods to modify the majority carriers in CNT- and graphene-based transistors such as chemical doping, oxygen doping, electrostatic doping, trap charge-induced doping, and metal work function engineering.
In order to have n-type conversion and p-type enhancement behavior in CNTs under ambient conditions, various chemical doping strategies have been investigated [171-179]. The choice of chemical dopant is complicated by the fact that the redox potential of CNTs is strongly diameter-dependent, as shown in Figure 9a [108]. The values in parentheses indicate the chiral index of the SWCNTs and the reduction potentials of dopants (BV, NADH, DDQ, $\mathrm{NOBF}_{4}$, and $\mathrm{AuCl}_{3}$ ) are also indicated as dotted lines. As shown in Figure $9 \mathrm{a}$, the $\mathrm{Au}^{3+}$ ion has the large reduction potential of $1.50 \mathrm{~V}$, which acts as p-type doping in CNTs. BV has an oxidation potential of $-1.1 \mathrm{~V}$, which implies that BV can act as an n-type dopants. BV donates electrons to the empty conduction band of semiconducting CNTs [180]. The right panel of Figure 9a shows an example of n-type CNT transistor by precisely positioning $\mathrm{BV}$ with inkjet printing on CNTs channel region [181]. Using $\beta$-nicotinamide adenine dinucleotide (reduced dipotassium salt, NADH), a type conversion in CNTs is also demonstrated distinctly [182]. A reduction potential of tetrafluorotetracyanop-quinodimethane (F4TCNQ) in the range of $0.1 \mathrm{~V}$ to $0.2 \mathrm{~V}$ makes it an electron extractor and p-type dopant [183]. For graphene, it has been demonstrated that the work function of CVD graphene can be modulated up to $1.1 \mathrm{eV}$ with BV doping [184]. Similarly, other work showed GO doping with Au allowed control of the work function [185]. For BLG, surface chemical doping in BLG can be utilized to induce a vertical displacement field. Interestingly, tunable Dirac points can be rationally controlled by the amount of BV doping, providing complementary inverter circuits [186]. Figure 9b shows a simple way to control polarity by just annealing the pCNT FET in vacuum, converting it to an $n$-CNT FET [187]. One of the reasons for having $\mathrm{p}$-character in CNT FETs is due to the interaction with $\mathrm{O}_{2}$ physisorbs on the CNT surface $[148,188,189]$. Originally a p-type CNT FET was converted to n-type after annealing process for removing $\mathrm{O}_{2}$ molecules [187]. It has been shown that the type conversion of CNT FETs could be possible by electrostatic doping using a charge-trap layer between the gate electrode and CNT channel [190,191]. Figure 9c shows the transfer characteristics of p-type and n-type CNT FETs converted using an Au floating gate. At high negative gate bias range, positive charges are trapped in the trap layer, and the threshold voltage is shifted in the negative bias direction. Therefore, the FETs show n-type characteristics in relatively small gate voltage sweep range. On the contrary, when high positive gate bias is initially applied, which traps the negative charges, the FETs show p-type characteristics in a relatively small gate voltage sweep range. Figure $9 d$ shows the electrical performance of an initially p-type characteristic as it is gradually changed to n-type via 

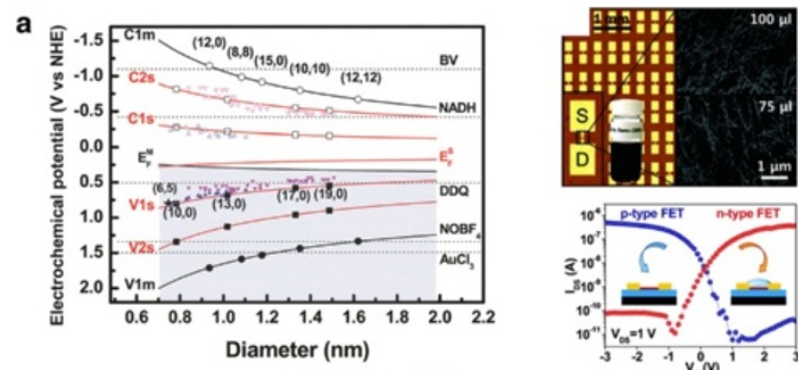

Chemical doping
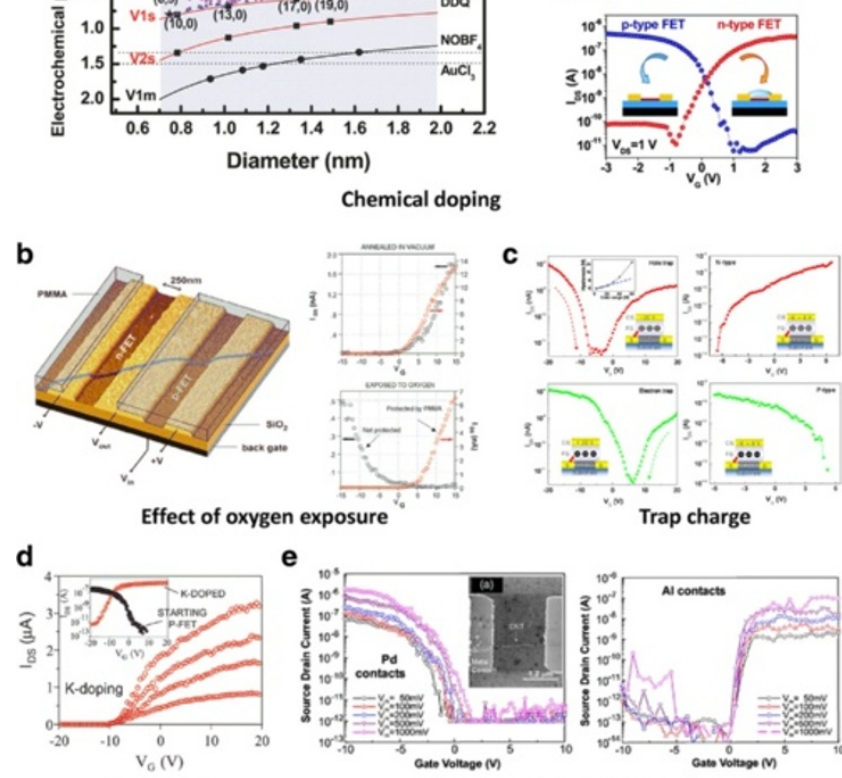

e

K - doping

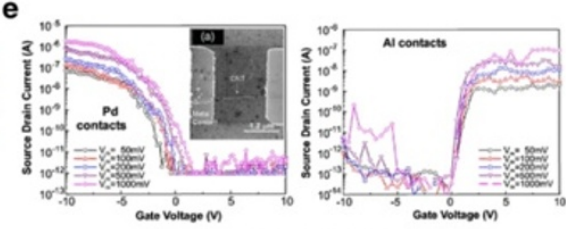

Contact work function

Figure 9 Various methods of polarity control of FETs based on CNTs and graphene. a, Redox potential of nanotubes as a function of the diameter (left). This Reproduced with permission [108]. Copyright 2010, Royal Society of Chemistry. Array of n-type CNT transistor by precisely positioning an air-stable BV. Reproduced with permission [181]. Copyright 2011, American Chemical Society. b, Effect of oxygen on p-doping. I-V curves of originally p-type CNT FET, with the nanotube capped with PMMA, have been converted to $n$-type. Reproduced with permission [187]. Copyright 2001, American Chemical Society. c, The type conversion of CNT FETs by trap layer-induced electrostatic doping. Adapted with permission [190]. d, I-V characteristic of an initially p-type characteristic in SWCNT FET, gradually changed to n-type caused by increasing amounts of K. Reproduced with permission [187]. Copyright 2001, American Chemical Society. e, Polarity control by metal (Pd and Al) work

function. Reproduced with permission [193]. Copyright 2005, American Institute of Physics.

increasing amounts of $\mathrm{K}$ on the nanotube [187,192]. Potassium ions have a high oxidation potential of $-0.7 \mathrm{~V}$ and act as an electron donor (n-type dopants) for CNTs. Logic circuits and pn junctions were fabricated by covering half of a CNT FET with PMMA and K-doping the exposed regions. The electrical polarity of SWCNT FETs can be affected by the work function of the contact metal, especially by the contact barrier control for the injection of carriers $[148,175,193]$. Figure 9e shows the transfer characteristics of CNT FETs using different metal contact electrodes such as Pd and Al [193]. The transfer characteristics show the presence of a p-type on-state but no n-branch in the case of high-work function metals such as $\mathrm{Pd}$ and $\mathrm{Ti}$, ambipolar behavior in the case of $\mathrm{Mg}$, and n-type only behavior in the case of Ca electrodes [194]. By varying the work function, the band alignment for a Mg-contacted device has efficient hole and electron injection, resulting in ambipolar characteristics. Conversely, due to work function and surface dipole formation, CNTs contacted by $\mathrm{Ca}$ electrodes have a suppressed p-type branch due to large energy barrier for holes. Although this method works to control the injection of carriers in single devices, the use of different metal electrodes in highdensity devices is commercially unreasonable and resulting devices still have highly variable contact properties.

Numerous efforts have been made to get higher on/off ratios and better control of carrier type in nano-carbon transistors. In order to understand advantages and disadvantages for CNT and graphene FETs, a side-by-side comparison is required. Table 2 shows the comparison for FET performance of CNTs and graphene devices. CNT FET and graphene devices exhibit output performances in a different manner. Moreover, the performances are distinct in different types of FET devices consisting of different forms of CNTs (single CNT, aligned CNT network, random CNT network) and graphene (CVD graphene, exfoliated BLG, GNR) with different gate structures. Nevertheless, a clear trade-off behavior between on/off ratio and mobility for each device was shown. 
Table 2 Device performance of various CNTs and graphene FETs

\begin{tabular}{|c|c|c|c|c|c|c|c|}
\hline Channel & Preparation method & $\begin{array}{c}\text { Transistor } \\
\text { structure }\end{array}$ & $\begin{array}{c}\text { Gate } \\
\text { dielectric }\end{array}$ & $\begin{array}{c}\text { Gate } \\
\text { length }(\mu \mathrm{m})\end{array}$ & Carrier type & $\begin{array}{c}\text { On/Off } \\
\text { ratio }\end{array}$ & $\begin{array}{l}\text { Mobility } \\
\left(\mathrm{cm}^{2} / \mathrm{Vs}\right)\end{array}$ \\
\hline Single CNT [32] & CVD on quartz & Back gate & $\mathrm{SiO}_{2}$ & 5 & p-type & $10^{5}$ & $636^{(C)}$ \\
\hline Aligned CNTs [32] & Electrical breakdown & Back gate & $\mathrm{HfO}_{2}$ & 12 & p-type & $2 \rightarrow 10^{4}$ & $570^{(C)} \rightarrow 200^{(C)}$ \\
\hline Random network CNTs [149] & Channel cutting & Top gate & $\mathrm{HfO}_{2}$ & 100 & p-type & $10 \rightarrow 10^{4}$ & $200^{(C)} \rightarrow 80^{(C)}$ \\
\hline Random network CNTs [153] & 97\% separated CNTs & Back gate & $\mathrm{SiO}_{2}$ & 20 & p-type & $10^{4}$ & $20^{(p)}$ \\
\hline Random network CNTs [181] & Viologen doped CNTs & Back gate & $\mathrm{HfO}_{2}$ & 9 & $p \rightarrow$ n-type & $10^{3}$ & $2^{(p)}$ \\
\hline Exfoliated graphene [141] & Monolayer graphene & Back gate & $\mathrm{SiO}_{2}$ & 4 & Ambipolar & 10 & $10,000^{(p)}$ \\
\hline CVD grown graphene [195] & Monolayer graphene & Back gate & $\mathrm{SiO}_{2}$ & 5 & Ambipolar & 10 & $1,100^{(p)}$ \\
\hline Exfoliated graphene [158] & Bilayer graphene & Dual gate & $\begin{array}{l}\mathrm{SiO}_{2} \text { (Back) } \\
\mathrm{HfO}_{2} \text { (Top) }\end{array}$ & 1.6 & Ambipolar & $5 \rightarrow 100$ & - \\
\hline Graphene nanoribbon [162] & $16 \rightarrow 6 \mathrm{~nm}$ nanoribbon & Back gate & $\mathrm{SiO}_{2}$ & 0.25 & Ambipolar $\rightarrow$ p-type & $1.5 \rightarrow 100$ & - \\
\hline
\end{tabular}

p: Parallel plate Model, c: Cylindrical Model, h: Hole Mobility, e: Electron Mobility.

\subsection{Flexible electronics}

\subsubsection{Integrated logic circuits}

Next-generation military and industrial radio-frequency (RF) surveillance systems will benefit from flexibility and stretchability of circuits for increased resilience. A realistic short- and medium-term goal for carbon electronics is utilizing the combination of electrical, mechanical, and optical properties of CNTs and graphene thin films to replace organic semiconductors and a-Si in these flexible/stretchable systems [196-204]. In this section, we introduce recent progress for integrating high-quality circuits on plastic substrates.

Figure 10a shows an integrated circuit fabricated with monolayer graphene as the electrodes and a SWCNT network for the channel [123]. Using this layout, transparent logic circuit arrays (inverters, NOR gates, and NAND gates) using SWCNT-channel/graphene-electrodes transistors were fabricated with a high yield of $80 \%$. The authors connected two p-type transistors to create a PMOS inverter with gain of approximately 1.4, with an operating voltage range of $0-5 \mathrm{~V}$. PMOS NOR and NAND logic gates were similarly constructed using three SWCNT/ graphene transistors. The graphene electrode and the SWCNT network channel are desirable not only for flexible and stretchable electronics, but also for use with invisible electronics due to the high transparency of atomically thin materials. Figure 10b shows a flexible four-bit row decoder circuit using SWCNT as the channel and metal electrodes [149]. A binary-encoded input of four data bits is successfully decoded using this decoder circuit. Due to the high mobility of the SWCNT thin films, even with critical dimensions $(100 \mu \mathrm{m})$ these decoder circuits can successfully operate in the $\mathrm{kHz}$ region. With such large channel lengths, cheap and scalable patterning methods such as screen printing are possible. More complex device structures are also easily possible such as master-slave delay flip-flops and 21-stage ring oscillators which were fabricated on PEN substrates [205]. Figure 10c demonstrates flexible complementary graphene inverters prepared on a plastic substrate by connecting two graphene transistors with a coplanar gate configuration. Fabrication was achieved using only two materials: graphene and an ion gel gate dielectric [206]. Unlike conventional solid state dielectrics, the operation of ion-gel gated transistors is based on the formation of a high capacitance electric double layer (EDL) under an electric field. The graphene inverter operates uniquely with two identical ambipolar transistors, unlike complementary inverters based on separate $\mathrm{n}$ - and p-channel transistors. Also in contrast to typical CMOS inverters, the output voltage did not saturate to zero or the supply voltage $\left(\mathrm{V}_{\mathrm{DD}}\right)$ due to the zero band gap of graphene [206]. With an estimated maximum voltage gain of 2.6, the technology is sufficient to drive subsequent components in logic circuits. Graphene-based frequency doublers and modulators on rigid substrates have been reported to demonstrate the feasible usage of graphene in analogue electronics [207-211]. Figure 10d shows a flexible all-graphene modulator circuit for quaternary digital modulations, which can encode two bits of information per symbol [212]. A couple of transistors are required for these two quaternary modulations.

\subsubsection{Other Flexible Applications}

Applications ranging from flexible solar cells, displays, e-papers, wearable and biomedical skin-like devices open up new opportunities in the field of electronics. In this section, we describe applications of several flexible devices possible with carbon electronics jsuch as sensors, LEDs, RF devices, stimulators, and memory devices.

As an example of further applications of flexible devices, Figure 11a demonstrates an active-matrix backplane for an artificial electronic skin (e-skin) device, capable of spatial touch mapping [213]. The SWCNT TFTs are used for a mechanically flexible backplane with polyimide as a 
a
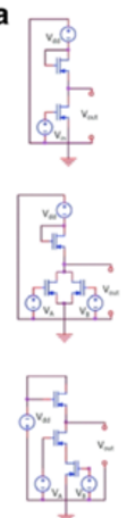

SWCNT inverter, NOR, NAND b
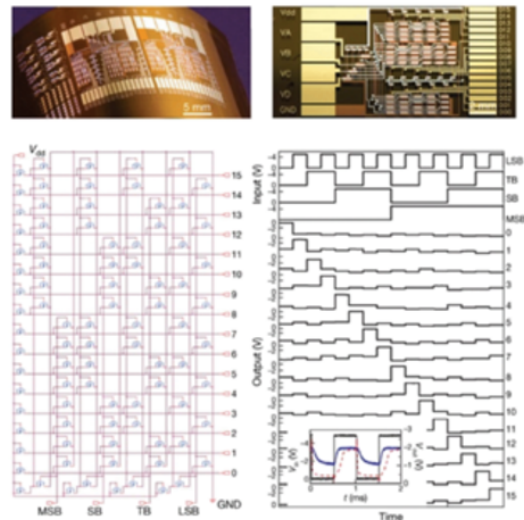

SWCNT decoder
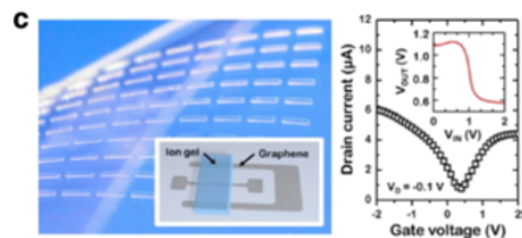

d
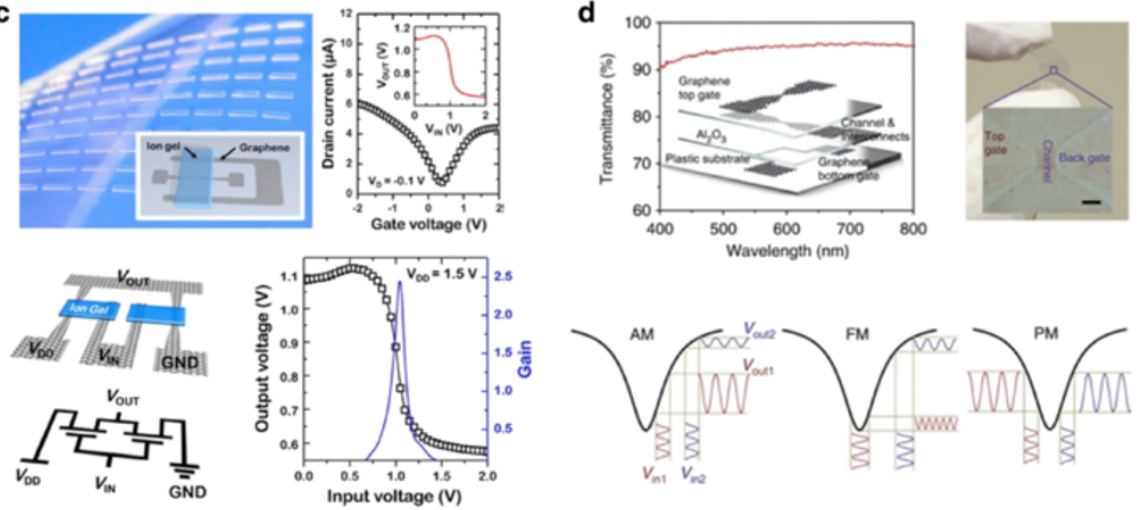

Graphene inverter

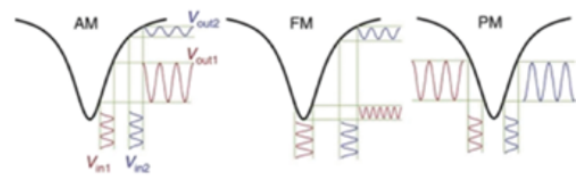

Graphene digital modulator

Figure 10 Flexible logic circuits using CNTs and graphene. a, Transparent and flexible logic circuits (inverter, NAND, and NOR) using graphene as electrodes and random network CNTs as the channel. Reproduced with permission [123]. Copyright 2011, American Chemical Society. b, Flexible four-bit row decoder circuit using SWCNT channel and metal electrodes. Reproduced with permission [149]. Copyright 2008, Nature Publishing Group. c, Flexible complementary graphene inverters prepared on plastic substrate with ion-gel gate dielectric. Reproduced with permission [206]. Copyright 2012, American Chemical Society. d, Transparent and flexible all-graphene digital modulator for quaternary digital modulations. Reproduced with permission [212]. Copyright 2012, Nature Publishing Group.

support substrate. The polyimide film substrate was utilized as a honeycomb mesh structure to make the substrate more robust against strain. Each pixel of pressure sensor is actively controlled by a SWCNT TFT. The sensor sensitivity shows $\sim 30 \mu \mathrm{SkPa}^{-1}$, which is three times larger than previous NW-based sensors [214]. Figure 11b shows the flexible active-matrix design with SWCNTs as the channel material. In these devices, high current drive is needed to actively switch OLEDs [215]. Each pixel is controlled by a SWCNT TFT that acts as a switch for an active-matrix of OLED and pressure sensor. Alternating current electroluminescence devices on flexible PET substrates were also demonstrated based on monolayer graphene electrodes [216]. Graphene seems to be an ideal material for high-speed systems owing to its extremely high carrier mobility. Despite poor switching behavior of graphene transistors limits their usage in digital/logic applications, they are still promising in the analogue/RF applications due to their atomic-thick layout that allows for shorter scaling of channel length. The combination of high speed and flexibility is a big challenge for flexible graphene RF devices [217-221]. RF devices using graphene have achieved cut-off frequencies between 100-300 GHz. Figure 11c shows the flexible solution-based graphene transistors at $\mathrm{GHz}$ frequencies with a current gain cut-off frequency of $2.2 \mathrm{GHz}$ and a power gain cut-off frequency of $550 \mathrm{MHz}$ [217]. Noninvasive probing and manipulation of biological tissue is another field where graphene is useful. Figure 11d reports a nonvascular surgical method to increase cerebral blood volume using a flexible, transparent, and biocompatible graphene electrical field stimulator [222]. The flexible graphene stimulator was placed onto the cortical brain without tissue damage or unnecessary neuronal activation. A noncontact electric field was 


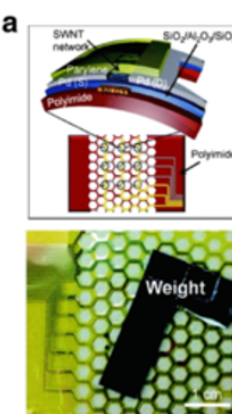

SWCNT pressure sensor

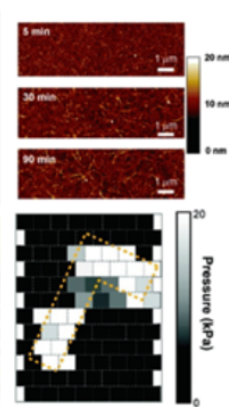

d c

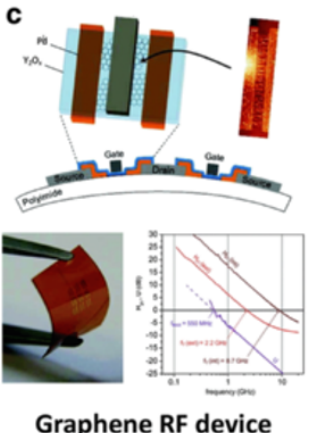

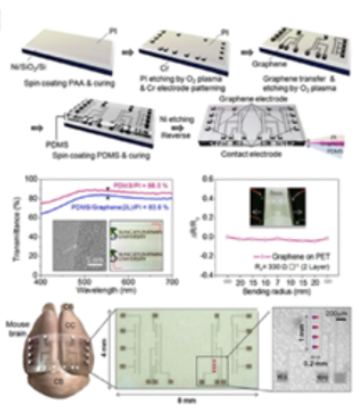

Graphene E - field stimulator b

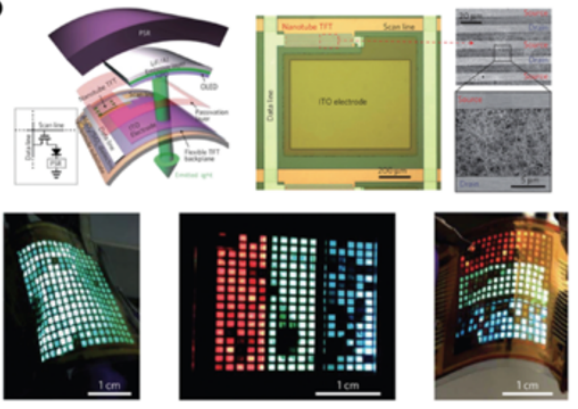

SWCNT LED

e

e
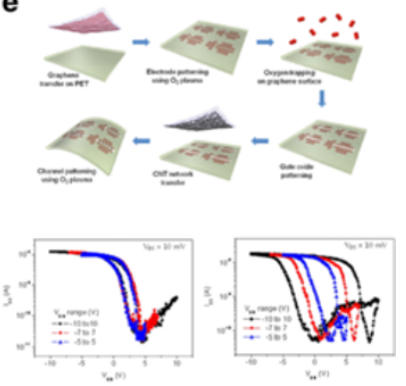

SWCNT/Graphene memory

Figure 11 Various flexible applications using CNTs and graphene. a, Active-matrix of SWCNT TFTs for a pressure sensor device. Reproduced with permission [213]. Copyright 2011, American Chemical Society. b. Flexible active-matrix design using SWCNTs as the channel material of the TFTs in OLEDs. Reproduced with permission [215]. Copyright 2013, Nature Publishing Group. c, Flexible RF device using solution-based graphene. Graphene is an ideal material for high-speed communication systems owing to its uniquely high carrier mobility. Reproduced with permission [217]. Copyright 2012, American Chemical Society. d, Flexible, transparent, and noncytotoxic graphene electric field stimulator. Reproduced with permission [222]. Copyright 2013, American Chemical Society. e, Transparent and flexible memory devices using SWCNT channel and graphene electrodes. The oxygen-decorated graphene electrode revealed an initially large hysteresis in SWCNT/graphene TFT. Adapted with permission [223].

applied at a specific local blood vessel to detect effective cerebral blood volume increases in mouse brains using in vivo optical recordings of signal imaging. In Figure 11e, transparent and flexible memory devices were fabricated using graphene electrodes and SWCNT channel [223]. The original electrical characteristics of the FET using graphene electrode without ozone treatment show small hysteresis. When the graphene gate was treated under an ozone generator, oxygen atoms and graphene have bonding as $\mathrm{C}-\mathrm{O}-\mathrm{C}, \mathrm{C}=\mathrm{O}$, and $\mathrm{C}-\mathrm{OH}$, which acted as charge trap sites. The FET with oxygen-decorated graphene electrode exhibits large hysteresis. This hysteresis-controllable FET can act as memory device, and showed no degradation of transmittance after oxygen decoration. This result is noticeable, compared to $\mathrm{Au}$ and $\mathrm{Al}$ nanoparticle trap layers that provided an $11.4 \%$ and $25 \%$ decrease in transmittance, respectively [224]. Flexible organic resistive memory devices with multilayer graphene electrodes were also reported [225]. Memory devices using a graphene oxide film were also fabricated on flexible substrates with reliable memory performance in terms of retention and endurance [226].

\subsection{Stretchable electronics}

Stretchability is a key parameter in the development of wearable devices that can be embedded into clothes and garments or even attached directly to the skin, where high levels of strain will be encountered. Possible applications include the human-friendly devices for detecting human motions, monitoring health system, and healing. In addition to flexibility, all these stretchable applications demand tolerance of large levels of strain $(>>1 \%)$ without fracture or significant degradation in electronic properties. The mainstream strategy to realize improved stretchability focuses on the development of stretchable materials including organic polymers, networks of 1-D wires, and nano-carbons [227-231]. Owing to the difficulties in developing new stretchable materials, geometrical engineering of the structures also needs to be addressed [6]. For example, ultrathin buckled geometries and pre-strained geometrically wavy materials offer stretchability with applied strain [232-235]. These devices can be integrated into larger systems containing conventional rigid materials. In this section, we introduce developed classes of material-based stretchable 
devices that use CNTs and graphene thin films on elastomer substrates.

\subsubsection{Stretchable conducting films}

Loading a SWCNT random network onto an elastomeric substrate simply affords a stretchable conducting film with the ability to accommodate strains greater than 20\% [236-238]. The left panel of Figure 12a shows transparent, conducting spray-deposited films of SWCNTs that can be stretched by applying strain along each axis [239]. This stretchable SWCNT film accommodates the stretchability by up to $150 \%$ with conductivities as high as $2,200 \mathrm{~S} \mathrm{~cm}^{-1}$ at the strain of $150 \%$. This property can be utilized to construct strain sensors, with performance comparable to conventional metal-strain gauges. Using a nonlinear buckling process as shown in the right panel of Figure 12a, ribbon arrays of CNT films can be modified into a "wavy" layout [231]. With a pre-strain (100\%) method, the wavy CNT ribbon can accommodate large stretching with the $4.1 \%$ resistance increases when the wavy $\mathrm{CNT}$ ribbon is stretched to the pre-strain stage. Applied strains lead to a reversible deformation of these buckled patterns which change the electrical properties. Together with the good optical and electrical properties, graphene films have excellent mechanical properties applicable to stretchable electrodes. One such example consists of few-layer CVD grown graphene films transferred onto elastic substrates, as shown in the left panel of Figure 12b [240]. The transferred film on an unstrained substrate recovers its original resistance after stretching by $\sim 6 \%$. In this work, the authors also transferred the film to pre-strained (12\%) substrates to enhance the electromechanical stabilities. Both longitudinal and transverse resistances $\left(\mathrm{R}_{\mathrm{y}}\right.$ and $\left.\mathrm{R}_{\mathrm{x}}\right)$ were stable up to $\sim 11 \%$ stretching with only one order of magnitude change at $\sim 25 \%$ stretching. $3 \mathrm{D}$-graphene macroscopic structures formed with a foamlike network of graphene was also developed using template-directed CVD (right panel of Figure 12b) [241]. The composites fabricated by this approach are a monolithic 3D-graphene network, in which electrical and mechanical properties were improved by using continuous CVD grown graphene building blocks. The results of graphene composites show stretchability over $50 \%$ with resistance changes stable after the fifth cycle of stretch-release.
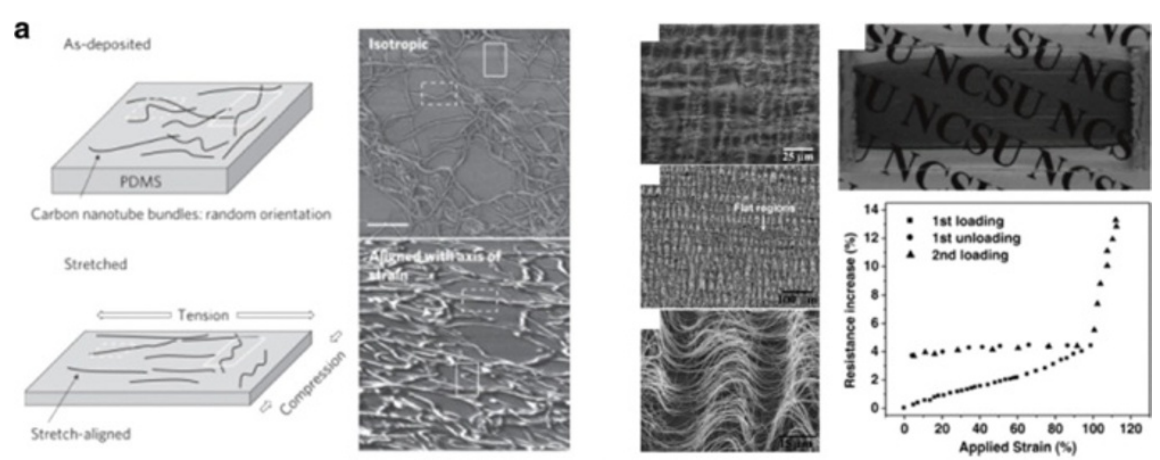

SWCNT conducting films
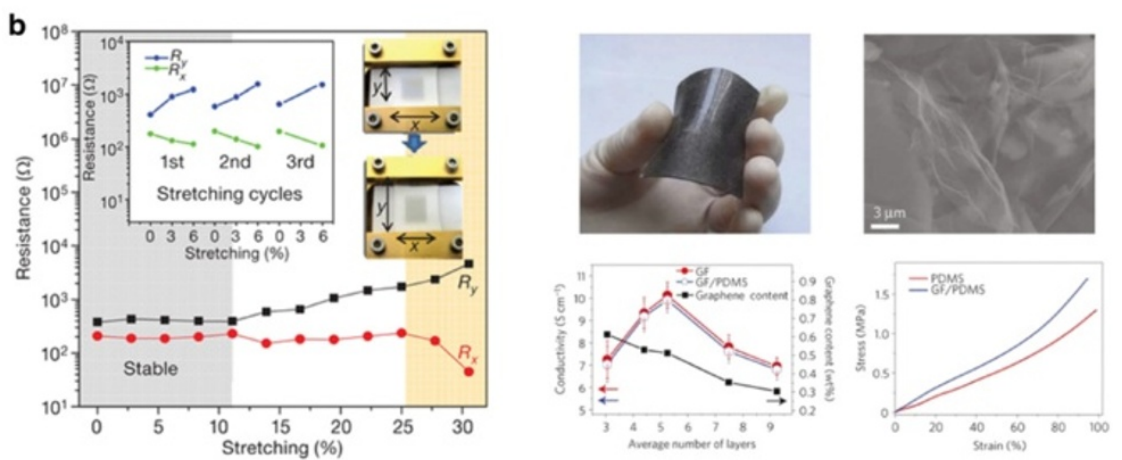

Graphene conducting films

Figure 12 Stretchable conducting films using CNTs and graphene. a, Transparent, conducting spray-deposited films of SWCNTs that can be rendered stretchable by applying strain along each axis (left). Reproduced with permission [239]. Copyright 2011, Nature Publishing Group. Wavy ribbons of CNTs are embedded in elastomeric substrates to fabricate stretchable conductors (right). Adapted with permission [231]. b, Stretchable conducting films using few-layer CVD grown graphene (left). 3D-graphene macroscopic structure with a foam-like network graphene (right). Reproduced with permission [240,241]. Copyright 2009 and 2011, Nature Publishing Group. 


\subsubsection{Stretchable applications}

Extreme difficulties are associated with the development of complete sets of stretchable electronics because all elements of the system need to be stretched out together. For instance, currently available carbon-based devices such as TFTs usually exhibit limited flexibility and stretchability owing to the use of fragile oxide dielectrics such as $\mathrm{Al}_{2} \mathrm{O}_{3}$ and $\mathrm{SiO}_{2}$. Polymer dielectrics have modest electrical performance despite their excellent bendability [242]. In this section, we introduce several strategies to fabricate stretchable devices using CNTs and graphene.

Reproduced with permission [246] Copyright 2011, Nature Publishing Group.

Figure 13a shows transparent and stretchable integrated circuits composed of CNTs and polymer dielectric [243]. The active channel and electrodes were all fabricated from CNTs (semiconducting and metallic), with PMMA dielectric layer and a plastic substrates. Although these were fabricated on plastic substrate, thermopressure was used for forming dorm-shape biaxial strain. The devices exhibit biaxial stretchability of up to $18 \%$ and the level of logic circuits include inverters, ring oscillators, NOR, NAND, XOR gates, and static random access memory (SRAM) cells. In Figure 13b, a graphene FET array on a stretchable rubber substrate with ion-gel dielectric is introduced [244]. Such all-graphene devices (graphene composes both the channel and electrodes) exhibit hole and electron mobilities of $\sim 1188$ and $\sim 422 \mathrm{~cm}^{2} \mathrm{~V}^{-1} \mathrm{~s}^{-1}$, respectively with stable operation up to $5 \%$ stretching. Although the stretchability of transistors is moderate, impressively the electrical properties were invariant even after 1000 cycles. Figure 13c shows a new approach for preparing a wrinkled gate dielectric using a transfer method to maximize the performance of the oxide without compromising the ability to stretch and bend [245]. A $50 \mathrm{~nm}$ aluminum oxide $\left(\mathrm{Al}_{2} \mathrm{O}_{3}\right)$ layer was deposited onto rough $\mathrm{Cu}$ foil using atomic layer deposition. After coating with PMMA, $\mathrm{Cu}$ foil was chemically etched, and the $\mathrm{Al}_{2} \mathrm{O}_{3}$ layer was then transferred as dielectric layer. This transferred $\mathrm{Al}_{2} \mathrm{O}_{3}$ layer was wrinkled with a "wavy" structure, which was robust under high tensile strain. The resulting TFTs exhibited device-acceptable electrical a
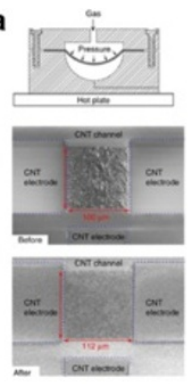

CNT transistor b

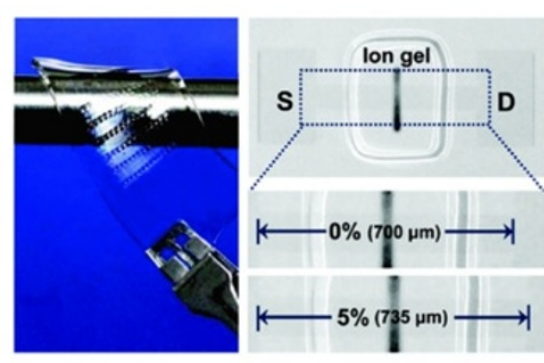

Graphene transistor

d

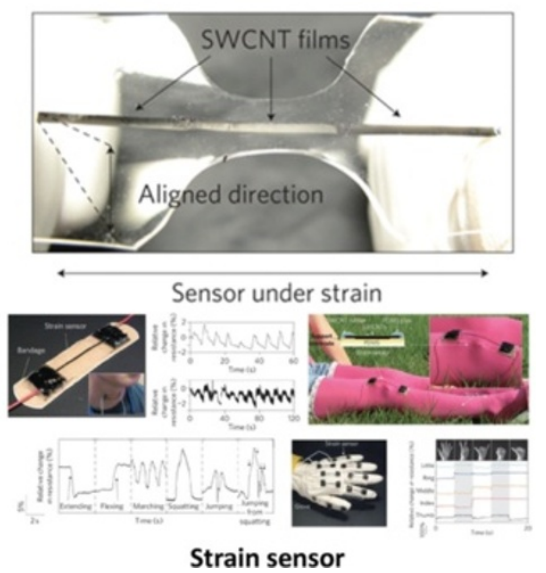

Figure 13 Stretchable applications using CNTs and graphene. a, Transparent and soft integrated circuits with random network SWCNT channel and PMMA dielectric layer on the PEN substrate. Reproduced with permission [243]. Copyright 2013, Nature Publishing Group. b, Graphene FET array on a stretchable rubber substrate with ion-gel dielectric. Reproduced with permission [244]. Copyright 2011, American Chemical Society. c, Stretchable and transparent TFTs combining SWCNTs/graphene with a geometrically wrinkled $\mathrm{Al}_{2} \mathrm{O}_{3}$ dielectric layer. Reproduced with permission [245]. Copyright 2013, Nature Publishing Group. d, Wearable and stretchable strain sensors fabricated from thin films of aligned SWCNTs. Reproduced with permission [246]. Copyright 2011, Nature Publishing Group. 
performance with small gate leakage current due to the build-in air gap between wrinkled $\mathrm{Al}_{2} \mathrm{O}_{3}$ and graphene gate. The devices were stretched along the length direction (16\% strain) and along the width direction (20\% strain), as shown in Figure 13c. The devices were stretched and released up to a maximum of 1,000 times without deterioration. Figure 13d shows a class of wearable and stretchable devices fabricated from thin films of aligned SWCNTs [246]. When stretched, the films fracture into gaps and islands with tube bundles bridging the gaps. This mechanism allows the films to act as strain sensors with capabilities extending up to $280 \%$ strain, which is 50 times more than conventional metal strain gauges, with high durability (10,000 cycles at $150 \%$ strain), and fast response (delay time of $14 \mathrm{~ms}$ ). When the CNT sensors were assembled on stockings, bandages and gloves to fabricate devices, the devices were able to detect human movement, typing, breathing and speech, each unique applications useful for developing humanfriendly and bio-integrated devices [239]. Figure 14 shows a summary of the flexible/stretchable device layouts and circuit levels of devices using nano-carbon, followed by the demonstrations of electrical, optical and mechanical properties.

\section{Conclusions}

\subsection{Summary and prospects}

We have reviewed the current status of CNTs and graphene in diverse applications of soft electronics from material preparation to performance in logic circuits. Lowdimensional carbon materials exhibit superb electronic properties and promising performance and are attractive for future electronics. Methods for synthesizing onedimensional CNT and two-dimensional graphene films, as

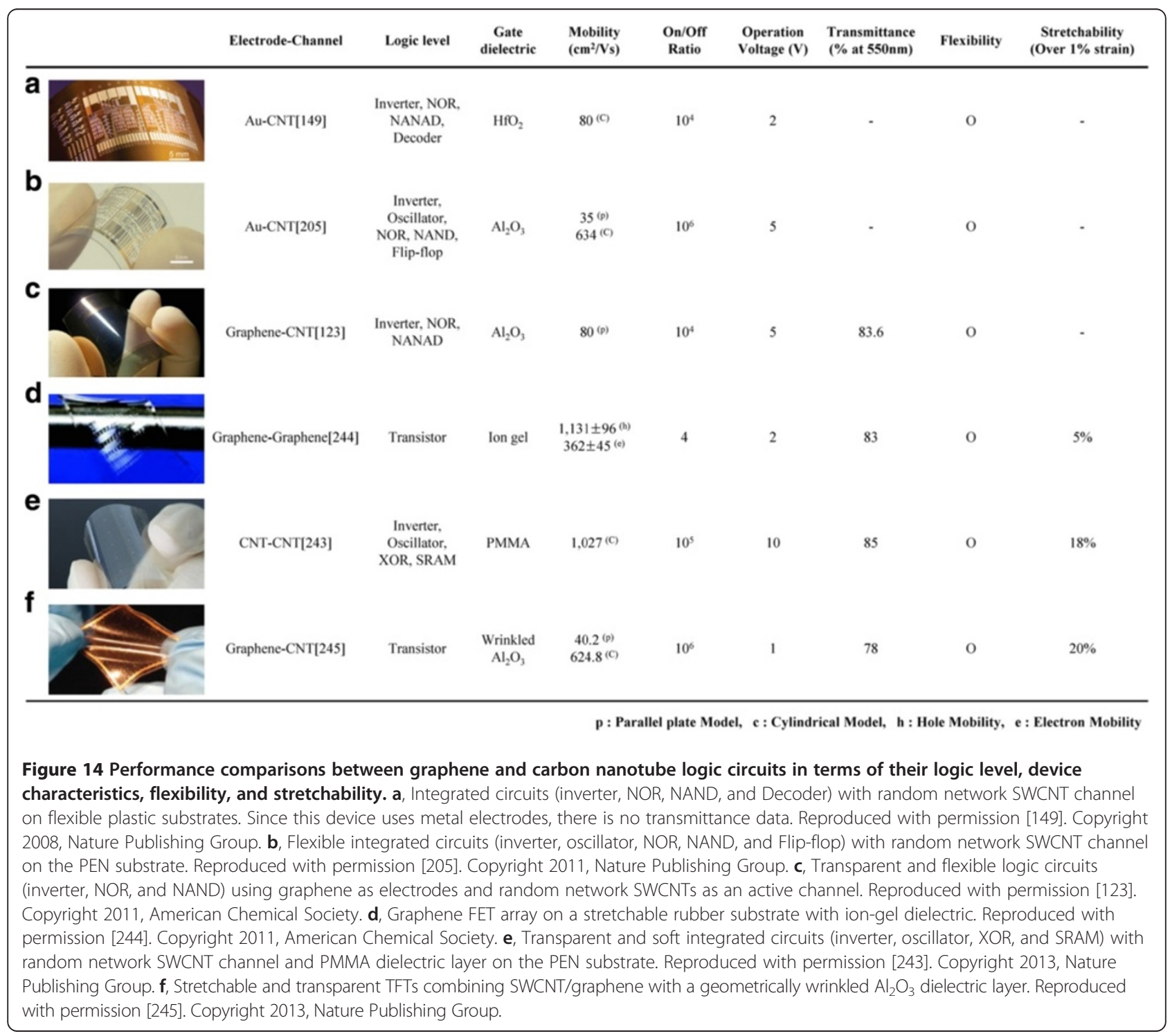


well as procedures for device fabrication on soft substrates have been discussed here. Both CNTs and graphene exemplify TCF properties including a high operational flexibility and stretchability that are not accessible with transparent ITO electrodes. Likewise, field effect mobilities of carbonbased transistors have reached levels unfeasible by organic semiconductors/a-Si. CNT FETs, whether composed of a single CNT, aligned CNTs, or random network CNTs, show high on/off ratio and mobility. Graphene FETs provide extremely high mobility but poor on/off ratio due to zero band gap. Engineering for on/off ratio increase and carrier polarity control were summarized. For applications in active electronics, SWCNT and graphene transistors can be assembled on a variety of substrates including flexible plastic and stretchable elastomers. Various complex integrated circuits based on nano-carbon materials have been demonstrated in the literature, as well. Each of these topics requires significant future exploration in order to realize commercialized applications of the immense potential of nano-carbon in next-generation electronics.

In spite of recent progress demonstrating the unique advantages of CNTs and graphene, the possible applications, social influence, addressable markets, and related economic issues will eventually decide the success of these nano-carbon materials. Both have unique and superb properties which open the possibility for soft electronics. Nevertheless, applications are limited by a different set of factors. Assemblies of CNTs are practical compared to the use of individual CNTs, but require the positioning of the CNTs in a specific direction, with desired density, and of desired metallicity/chirality. Methods to achieve this control are a current hot topic, but adoption of a particular method will require a high yield for industrial utilization even in niche applications. Conversely, graphene can be prepared in a large-area format. Yet, the transfer to a desired substrate may provoke damage in the graphene layer and degrade device performance. Therefore, developing a smart way of assembling CNTs to maximize the device performance and robust method of transfer of large-area graphene are two key ingredients that are unsolved but required for application. On a systems level, future electronics including biomedical applications with biocompatibility will require further research. For instance, CNTs and graphene combine synergistically, showing better flexibility and stretchability with no degradation of electrical performance when engineered to maximize potential. Additionally, combining both stretchable materials and stretchable geometries can allow for extremely stretchable systems. Aside from the engineering challenges of applying nano-carbon to soft electronics, CNTs and graphene are outstanding materials for demonstrating a number of basic science concepts in the fields of quantum electrodynamics, quantum optics, and quantum chemistry. Controlled synthesis and application of monolayer materials also allows exploration into a new class of vertical tunneling devices. Aside from carbon, other classes of graphene-like 2D materials such as transition-metal dichalcogenide (TMD) materials and boron nitride $(\mathrm{BN})$, might also be promising in the field of soft electronics when a band gap or other electrical/ mechanical properties are required. These related engineering opportunities in areas with the broad range of influential research topics provides strong motivation for continued efforts in human-friendly soft electronics.

\section{Competing interests}

The authors declare no competing financial interests.

\section{Authors' contributions}

SHC and YHL contributed to this work in the manuscript preparation. Both authors read and approved the final manuscript.

\section{Authors' information}

Sang Hoon Chae: Sang Hoon Chae is a researcher of the Center for Integrated Nanostructure Physics (CINAP), Institute of Basic Science (IBS) in Korea. He is a Ph.D. candidate within the Department of Energy Science at Sungkyunkwan University. He received his B.Sc. in the Department of Semiconductor Systems Engineering from Sungkyunkwan University, Korea, in 2010. His research interests include flexible and stretchable electronics using carbon nanotubes, graphene, and other 2D materials. Young Hee Lee: Young Hee Lee is a Director of the Center for Integrated Nanostructure Physics (CINAP), Institute of Basic Science (IBS) in Korea. He is a professor in the Department of Energy Science and Physics at Sungkyunkwan University, Korea. He received his B.Sc. in physics from Chonbuk National University, Korea and his Ph.D. in physics from Kent State University, USA. His research focus include fundamental studies of nanomaterials and their applications to electronic and optical devices, energy harvesting, and nanobiomedical areas: transparent, flexible, and stretchable transistors, supercapacitors, nanobatteries, hydrogen storage, neuroscience, and cancer therapy using noncontact electrical stimulators.

\section{Acknowledgements}

This work was supported by the Institute for Basic Science (IBS) and in part by BK-Plus through Ministry of Education, Korea. We thank David Perello (CINAP) and Alex Patterson (MIT) for valuable discussions during the preparation of this manuscript.

Received: 16 January 2014 Accepted: 4 March 2014

Published online: 25 April 2014

\section{References}

1. HJ Leamy, JH Wernick, Semiconductor silicon: the extraordinary made ordinary. MRS Bull. 22, 47-55 (1997)

2. HR Huff, An electronics division retrospective 1952-2002 and future opportunities in the twenty-first century. J. Electrochem. Soc. 149, S35-S58 (2002). doi:10.1149/1.1471893

3. TP Morgan, Intel Xeon Phi Battles GPUs, Defends x86 in Supercomputers, 2012. http://www.theregister.co.uk/2012/11/12/intel_xeon_phi_ coprocessor launch/

4. JA Rogers, T Someya, Y Huang, Materials and mechanics for stretchable electronics. Science 327, 1603-1607 (2010). doi:10.1126/science.1182383

5. T Someya, Y Kato, T Sekitani, S Iba, Y Noguchi, Y Murase, H Kawaguchi, T Sakurai, Conformable, flexible, large-area networks of pressure and thermal sensors with organic transistor active matrixes. Proc. Natl. Acad. Sci. U. S. A. 102, 12321-12325 (2005). doi:10.1073/pnas.0502392102

6. D-H Kim, JA Rogers, Stretchable electronics: materials strategies and devices. Adv. Mater. 20, 4887-4892 (2008). doi:10.1002/adma.200801788

7. A Shah, J Meier, A Buechel, U Kroll, J Steinhauser, F Meillaud, H Schade, D Dominé, Towards very low-cost mass production of thin-film silicon photovoltaic (PV) solar modules on glass. Thin Solid Films 502, 292-299 (2006). doi:10.1016/j.tsf.2005.07.299 
8. MD Jacunski, MS Shur, M Hack, Threshold voltage, field effect mobility, and gate-to-channel capacitance in polysilicon TFT's. IEEE Trans. Electron. Devices. 43, 1433-1440 (1996). doi:10.1109/16.535329

9. DH Oh, YH Lee, Stability and cap formation mechanism of single-walled carbon nanotubes. Phys. Rev. B 58, 7407-7411 (1998). doi:10.1103/ PhysRevB.58.7407

10. E Artukovic, M Kaempgen, DS Hecht, S Roth, G GrUner, Transparent and flexible carbon nanotube transistors. Nano Lett. 5, 757-760 (2005). doi:10.1021/nl050254o

11. L Hu, W Yuan, P Brochu, G Gruner, Q Pei, Highly stretchable, conductive, and transparent nanotube thin films. Appl. Phys. Lett. 94, 161108 (2009). doi:10.1063/1.3114463

12. Q Liu, Z Liu, X Zhang, L Yang, N Zhang, G Pan, S Yin, Y Chen, J Wei, Polymer photovoltaic cells based on solution-processable graphene and P3HT. Adv. Funct. Mater. 19, 894-904 (2009). doi:10.1002/adfm.200800954

13. T Durkop, SA Getty, E Cobas, MS Fuhrer, Extraordinary mobility in semiconducting carbon nanotubes. Nano Lett. 4, 35-39 (2004). doi:10.1021/ nl034841q

14. AS Mayorov, RV Gorbachev, SV Morozov, L Britnell, R Jalil, LA Ponomarenko, P Blake, KS Novoselov, K Watanabe, T Taniguchi, AK Geim, Micrometer-scale ballistic transport in encapsulated graphene at room temperature. Nano letters. 11, 2396-2399 (2011). doi:10.1021/nl200758b

15. KI Bolotin, KJ Sikes, Z Jiang, M Klima, G Fudenberg, J Hone, P Kim, HL Stormer, Ultrahigh electron mobility in suspended graphene. Solid State Commun. 146, 351-355 (2008). doi:10.1016/j.ssc.2008.02.024

16. A Javey, P Qi, Q Wang, H Dai, Ten- to 50-nm-long quasi-ballistic carbon nanotube devices obtained without complex lithography. Proc. Natl. Acad. Sci. U. S. A. 101, 13408-13410 (2004). doi:10.1073/pnas.0404450101

17. J Yu, G Liu, AV Sumant, V Goyal, AA Balandin, Graphene-on-diamond devices with increased current-carrying capacity: carbon sp2-on-sp3 technology. Nano letters. 12, 1603-1608 (2012). doi:10.1021/n1204545a

18. E Pop, D Mann, Q Wang, KE Goodson, HJ Dai, Thermal conductance of an individual single-wall carbon nanotube above room temperature. Nano Lett. 6, 96-100 (2006). doi:10.1021/nl052145f

19. AA Balandin, S Ghosh, W Bao, I Calizo, D Teweldebrhan, F Miao, CN Lau, Superior thermal conductivity of single-layer graphene. Nano Lett. 8, 902-907 (2008). doi:10.1021/nl0731872

20. RR Nair, P Blake, AN Grigorenko, KS Novoselov, TJ Booth, T Stauber, NMR Peres, AK Geim, Fine structure constant defines visual transparency of graphene. Science 320, 1308-1308 (2008). doi:10.1126/science.1156965

21. C Lee, X Wei, JW Kysar, J Hone, Measurement of the elastic properties and intrinsic strength of monolayer graphene. Science 321, 385-388 (2008). doi:10.1126/science.1157996

22. JU Park, MA Meitl, SH Hur, ML Usrey, MS Strano, PJ Kenis, JA Rogers, In situ deposition and patterning of single-walled carbon nanotubes by laminar flow and controlled flocculation in microfluidic channels. Angew. Chem. 45, 581-585 (2006). doi:10.1002/anie.200501799

23. MA Meitl, YX Zhou, A Gaur, S Jeon, ML Usrey, MS Strano, JA Rogers, Solution casting and transfer printing single-walled carbon nanotube films. Nano Lett. 4, 1643-1647 (2004). doi:10.1021/nl0491935

24. JM Moon, KH An, YH Lee, YS Park, DJ Bae, GS Park, High-yield purification process of singlewalled carbon nanotubes. J. Phys. Chem. B 105, 5677-5681 (2001). doi:10.1021/jp0102365

25. R Vajtai, BQ Wei, PM Ajayan, Controlled growth of carbon nanotubes. Philos. Trans. R. Soc. A-Math. Phys. Eng. Sci. 362, 2143-2160 (2004). doi:10.1098/ rsta.2004.1433

26. B Kitiyanan, WE Alvarez, JH Harwell, DE Resasco, Controlled production of single-wall carbon nanotubes by catalytic decomposition of $\mathrm{CO}$ on bimetallic Co-Mo catalysts. Chem. Phys. Lett. 317, 497-503 (2000). doi:10.1016/s0009-2614(99)01379-2

27. A Thess, R Lee, P Nikolaev, HJ Dai, P Petit, J Robert, CH Xu, YH Lee, SG Kim, AG Rinzler, DT Colbert, GE Scuseria, D Tomanek, JE Fischer, RE Smalley, Crystalline ropes of metallic carbon nanotubes. Science 273, 483-487 (1996). doi:10.1126/science.273.5274.483

28. MJ Bronikowski, PA Willis, DT Colbert, KA Smith, RE Smalley, Gas-phase production of carbon single-walled nanotubes from carbon monoxide via the HiPco process: A parametric study. J. Vac. Sci. Technol. A: Vac, Surf. Films. 19, 1800 (2001). doi:10.1116/1.1380721

29. Z Jin, H Chu, J Wang, J Hong, W Tan, Y Li, Ultralow feeding gas flow guiding growth of large-scale horizontally aligned single-walled carbon nanotube arrays. Nano Lett. 7, 2073-2079 (2007). doi:10.1021/nl070980m
30. W Zhou, Z Han, J Wang, Y Zhang, Z Jin, X Sun, Y Zhang, C Yan, Y Li, Copper catalyzing growth of single-walled carbon nanotubes on substrates. Nano Lett. 6, 2987-2990 (2006). doi:10.1021/nl061871v

31. BH Hong, JY Lee, T Beetz, YM Zhu, P Kim, KS Kim, Quasi-continuous growth of ultralong carbon nanotube arrays. J. Am. Chem. Soc. 127, 15336-15337 (2005). doi:10.1021/ja054454d

32. SJ Kang, C Kocabas, T Ozel, M Shim, N Pimparkar, MA Alam, SV Rotkin, JA Rogers, High-performance electronics using dense, perfectly aligned arrays of single-walled carbon nanotubes. Nature nanotechnology. 2, 230-236 (2007). doi:10.1038/nnano.2007.77

33. I-H Lee, J-W Im, U-J Kim, E-J Bae, K-K Kim, E-H Lee, Y-H Lee, S-H Hong, Y-S Min, Low temperature growth of single-walled carbon nanotube forest. Bull, Kor. Chem. Soc. 31, 2819-2822 (2010). doi:10.5012/bkcs.2010.31.10.2819

34. K Hata, DN Futaba, K Mizuno, T Namai, M Yumura, S lijima, Water-assisted highly efficient synthesis of impurity-free single-walled carbon nanotubes. Science 306, 1362-1364 (2004). doi:10.1126/science.1104962

35. ZF Ren, Synthesis of large arrays of well-aligned carbon nanotubes on glass. Science 282, 1105-1107 (1998). doi:10.1126/science.282.5391.1105

36. UJ Kim, IH Lee, JJ Bae, S Lee, GH Han, SJ Chae, F Guenes, JH Choi, CW Baik, SI Kim, JM Kim, YH Lee, Graphene/Carbon nanotube hybrid-based transparent 2D optical array. Adv. Mater. 23, 3809-+ (2011). doi:10.1002/ adma.201101622

37. D Wang, P Song, C Liu, W Wu, S Fan, Highly oriented carbon nanotube papers made of aligned carbon nanotubes. Nanotechnology 19, 075609 (2008). doi:10.1088/0957-4484/19/7/075609

38. SC Lim, K Lee, IH Lee, YH Lee, Field emission and application of carbon nanotubes. Nano 2, 69-89 (2007)

39. SC Lim, HK Choi, HJ Jeong, YI Song, GY Kim, KT Jung, YH Lee, A strategy for forming robust adhesion with the substrate in a carbon-nanotube fieldemission array. Carbon 44, 2809-2815 (2006). doi:10.1016/j.carbon.2006.03.030

40. L Hu, DS Hecht, G Gruner, Percolation in transparent and conducting carbon nanotube networks. Nano Lett. 4, 2513-2517 (2004). doi:10.1021/ nl048435y

41. A Moisala, AG Nasibulin, El Kauppinen, The role of metal nanoparticles in the catalytic production of single-walled carbon nanotubes - a review. J. Phys-Condense Matter. 15, S3011-S3035 (2003). doi:10.1088/0953-8984/ $15 / 42 / 003$

42. RC Tenent, TM Barnes, JD Bergeson, AJ Ferguson, B To, LM Gedvilas, MJ Heben, JL Blackburn, Ultrasmooth, large-area, high-uniformity, conductive transparent single-walled-carbon-nanotube films for photovoltaics produced by ultrasonic spraying. Adv. Mater. 21, 3210-3216 (2009). doi:10.1002/ adma.200803551

43. H-Z Geng, KK Kim, KP So, YS Lee, Y Chang, YH Lee, Effect of acid treatment on carbon nanotube-based flexible transparent conducting films. J. Am. Chem. Soc. 129, 7758-+ (2007). doi:10.1021/ja0722224

44. Z Wu, Z Chen, X Du, JM Logan, J Sippel, M Nikolou, K Kamaras, JR Reynolds, DB Tanner, AF Hebard, AG Rinzler, Transparent, conductive carbon nanotube films. Science 305, 1273-1276 (2004). doi:10.1126/ science. 1101243

45. W Jong Yu, S Yol Jeong, K Kang Kim, B Ram Kang, D Jae Bae, M Lee, S Hong, S Prabhu Gaunkar, D Pribat, D Perello, M Yun, J-Y Choi, Y Hee Lee, Bias-induced doping engineering with ionic adsorbates on single-walled carbon nanotube thin film transistors. New J. Phys. 10, 113013 (2008). doi:10.1088/1367-2630/10/11/113013

46. K Kordas, T Mustonen, G Toth, H Jantunen, M Lajunen, C Soldano, S Talapatra, S Kar, R Vajtai, PM Ajayan, Inkjet printing of electrically conductive patterns of carbon nanotubes. Small 2, 1021-1025 (2006). doi:10.1002/ smll.200600061

47. C Biswas, YH Lee, Graphene versus carbon nanotubes in electronic devices. Adv. Funct. Mater. 21, 3806-3826 (2011). doi:10.1002/adfm.201101241

48. YX Zhou, A Gaur, SH Hur, C Kocabas, MA Meitl, M Shim, JA Rogers, pchannel, $n$-channel thin film transistors and $\mathrm{p}-\mathrm{n}$ diodes based on single wall carbon nanotube networks. Nano Lett. 4, 2031-2035 (2004). doi:10.1021/ nl048905o

49. M Glerup, J Steinmetz, D Samaille, O Stéphan, S Enouz, A Loiseau, S Roth, P Bernier, Synthesis of N-doped SWNT using the arc-discharge procedure. Chem. Phys. Lett. 387, 193-197 (2004). doi:10.1016/j.cplett.2004.02.005

50. G Zhang, D Mann, L Zhang, A Javey, Y Li, E Yenilmez, Q Wang, JP McVittie, Y Nishi, J Gibbons, H Dai, Ultra-high-yield growth of vertical single-walled carbon nanotubes: hidden roles of hydrogen and oxygen. Proc. Natl. Acad. Sci. U. S. A. 102, 16141-16145 (2005). doi:10.1073/pnas.0507064102 
51. M Zhang, KR Atkinson, RH Baughman, Multifunctional carbon nanotube yarns by downsizing an ancient technology. Science 306, 1358-1361 (2004). doi:10.1126/science.1104276

52. K Liu, Y Sun, R Zhou, H Zhu, J Wang, L Liu, S Fan, K Jiang, Carbon nanotube yarns with high tensile strength made by a twisting and shrinking method. Nanotechnology 21, 045708 (2010). doi:10.1088/09574484/21/4/045708

53. X Zhang, K Jiang, C Feng, P Liu, L Zhang, J Kong, T Zhang, Q Li, S Fan, Spinning and processing continuous yarns from 4-inch wafer scale superaligned carbon nanotube arrays. Adv. Mater. 18, 1505-1510 (2006) doi:10.1002/adma.200502528

54. K Jiang, J Wang, Q Li, L Liu, C Liu, S Fan, Superaligned carbon nanotube arrays, films, and yarns: a road to applications. Adv. Mater. 23, 1154-1161 (2011). doi:10.1002/adma.201003989

55. KS Novoselov, AK Geim, SV Morozov, D Jiang, Y Zhang, SV Dubonos, IV Grigorieva, AA Firsov, Electric field effect in atomically thin carbon films. Science 306, 666-669 (2004). doi:10.1126/science.1102896

56. KS Novoselov, AK Geim, SV Morozov, D Jiang, MI Katsnelson, IV Grigorieva, SV Dubonos, AA Firsov, Two-dimensional gas of massless Dirac fermions in graphene. Nature 438, 197-200 (2005). doi:10.1038/nature04233

57. SJ Chae, F Güneş, KK Kim, ES Kim, GH Han, SM Kim, H-J Shin, S-M Yoon, J-Y Choi, MH Park, CW Yang, D Pribat, YH Lee, Synthesis of Large-Area Graphene Layers on Poly-Nickel Substrate by Chemical Vapor Deposition: Wrinkle Formation. Adv. Mater. 21, 2328-2333 (2009). doi:10.1002/adma.200803016

58. A Reina, X Jia, J Ho, D Nezich, H Son, V Bulovic, MS Dresselhaus, J Kong, Large area, few-layer graphene films on arbitrary substrates by chemical vapor deposition. Nano Lett. 9, 30-35 (2009). doi:10.1021/nl801827v

59. GH Han, SJ Chae, ES Kim, F Guenes, IH Lee, SW Lee, SY Lee, SC Lim, HK Jeong, MS Jeong, YH Lee, Laser thinning for monolayer graphene formation: heat sink and interference effect. Acs Nano. 5, 263-268 (2011). doi:10.1021/nn1026438

60. GH Han, F Gunes, JJ Bae, ES Kim, SJ Chae, HJ Shin, JY Choi, D Pribat, YH Lee, Influence of copper morphology in forming nucleation seeds for graphene growth. Nano letters. 11, 4144-4148 (2011). doi:10.1021/nl201980p

61. DL Duong, GH Han, SM Lee, F Gunes, ES Kim, ST Kim, H Kim, QH Ta, KP So, SJ Yoon, SJ Chae, YW Jo, MH Park, SH Chae, SC Lim, JY Choi, YH Lee, Probing graphene grain boundaries with optical microscopy. Nature 490, 235-239 (2012). doi:10.1038/nature11562

62. TH Ly, DL Duong, QH Ta, F Yao, QA Vu, HY Jeong, SH Chae, YH Lee, Nondestructive characterization of graphene defects. Adv. Funct. Mater. 23, 5183-5189 (2013). doi:10.1002/adfm.201300493

63. Y Hernandez, V Nicolosi, M Lotya, FM Blighe, Z Sun, S De, IT McGovern, B Holland, M Byrne, YK Gun'Ko, JJ Boland, P Niraj, G Duesberg, S Krishnamurthy, R Goodhue, J Hutchison, V Scardaci, AC Ferrari, JN Coleman, High-yield production of graphene by liquid-phase exfoliation of graphite. Nature nanotechnology. 3, 563-568 (2008). doi:10.1038/nnano.2008.215

64. X Wang, L Zhi, K Muellen, Transparent, conductive graphene electrodes for dye-sensitized solar cells. Nano Lett. 8, 323-327 (2008). doi:10.1021/ nl072838r

65. C Mattevi, G Eda, S Agnoli, S Miller, KA Mkhoyan, O Celik, D Mastrogiovanni, G Granozzi, E Garfunkel, M Chhowalla, Evolution of electrical, chemical, and structural properties of transparent and conducting chemically derived graphene thin films. Adv. Funct. Mater. 19, 2577-2583 (2009). doi:10.1002/ adfm.200900166

66. G Eda, G Fanchini, M Chhowalla, Large-area ultrathin films of reduced graphene oxide as a transparent and flexible electronic material. Nat. Nanotechnol. 3, 270-274 (2008). doi:10.1038/nnano.2008.83

67. M Jin, H-K Jeong, T-H Kim, KP So, Y Cui, WJ Yu, EJ Ra, YH Lee, Synthesis and systematic characterization of functionalized graphene sheets generated by thermal exfoliation at low temperature. J. Phys. D. Appl. Phys. 43, 275402 (2010). doi:10.1088/0022-3727/43/27/275402

68. H-K Jeong, M Jin, EJ Ra, KY Sheem, GH Han, S Arepalli, YH Lee, Enhanced electric double layer capacitance of graphite oxide intercalated by poly (sodium 4-styrensulfonate) with high cycle stability. Acs Nano. 4, 1162-1166 (2010). doi:10.1021/nn901790f

69. H-K Jeong, L Colakerol, MH Jin, P-A Glans, KE Smith, YH Lee, Unoccupied electronic states in graphite oxides. Chem. Phys. Lett. 460, 499-502 (2008). doi:10.1016/j.cplett.2008.06.042

70. H-K Jeong, YP Lee, MH Jin, ES Kim, JJ Bae, YH Lee, Thermal stability of graphite oxide. Chem. Phys. Lett. 470, 255-258 (2009). doi:10.1016/ j.cplett.2009.01.050
71. H-K Jeong, MH Jin, $\mathrm{KH}$ An, YH Lee, Structural stability and variable dielectric constant in poly sodium 4-styrensulfonate intercalated graphite oxide. J. Phys. Chem. C 113, 13060-13064 (2009). doi:10.1021/jp9026282

72. H-J Shin, KK Kim, A Benayad, S-M Yoon, HK Park, I-S Jung, MH Jin, H-K Jeong, JM Kim, J-Y Choi, YH Lee, Efficient reduction of graphite oxide by sodium borohydride and its effect on electrical conductance. Adv. Funct. Mater. 19, 1987-1992 (2009). doi:10.1002/adfm.200900167

73. M Jin, TH Kim, SC Lim, DL Duong, HJ Shin, YW Jo, HK Jeong, J Chang, S Xie, YH Lee, Facile physical route to highly crystalline graphene. Adv. Funct. Mater. 21, 3496-3501 (2011). doi:10.1002/adfm.201101037

74. KV Emtsev, A Bostwick, K Horn, J Jobst, GL Kellogg, L Ley, JL McChesney, T Ohta, SA Reshanov, J Rohrl, E Rotenberg, AK Schmid, D Waldmann, HB Weber, T Seyller, Towards wafer-size graphene layers by atmospheric pressure graphitization of silicon carbide. Nat. Mat. 8, 203-207 (2009). doi:10.1038/nmat2382

75. A Ouerghi, MG Silly, M Marangolo, C Mathieu, M Eddrief, M Picher, F Sirotti, S El Moussaoui, R Belkhou, Large-area and high-quality epitaxial graphene on off-axis SiC wafers. Acs Nano. 6, 6075-6082 (2012). doi:10.1021/ $\mathrm{nn} 301152 \mathrm{p}$

76. JS Moon, D Curtis, M Hu, D Wong, C McGuire, PM Campbell, G Jernigan, JL Tedesco, B VanMil, R Myers-Ward, C Eddy Jr, DK Gaskill, RF EpitaxialGraphene, Field-effect transistors on Si-Face 6H-SiC substrates. IEEE Electr. Device L. 30, 650-652 (2009). doi:10.1109/led.2009.2020699

77. Y Wu, YM Lin, AA Bol, KA Jenkins, F Xia, DB Farmer, Y Zhu, P Avouris, High-frequency, scaled graphene transistors on diamond-like carbon. Nature 472, 74-78 (2011). doi:10.1038/nature09979

78. J Hackley, D Ali, J DiPasquale, JD Demaree, CJK Richardson, Graphitic carbon growth on Si(111) using solid source molecular beam epitaxy. Appl. Phys. Lett. 95, 133114 (2009). doi:10.1063/1.3242029

79. YH Lee, L Yu, H Wang, W Fang, X Ling, Y Shi, CT Lin, JK Huang, MT Chang, CS Chang, M Dresselhaus, T Palacios, LJ Li, J Kong, Synthesis and transfer of single-layer transition metal disulfides on diverse surfaces. Nano letters. 13, 1852-1857 (2013). doi:10.1021/n|400687n

80. X Li, Y Zhu, W Cai, M Borysiak, B Han, D Chen, RD Piner, L Colombo, RS Ruoff, Transfer of large-area graphene films for high-performance transparent conductive electrodes. Nano Lett. 9, 4359-4363 (2009). doi:10.1021/nl902623y

81. S-H Hur, OO Park, JA Rogers, Extreme bendability of single-walled carbon nanotube networks transferred from high-temperature growth substrates to plastic and their use in thin-film transistors. Appl. Phys. Lett. 86, 243502 (2005). doi:10.1063/1.1947380

82. GH Han, H-J Shin, ES Kim, SJ Chae, J-Y Choi, YH Lee, Poly(Ethylene Co-Vinyl Acetate)-Assisted one-step transfer of ultra-large graphene. Nano 06, 59-65 (2011). doi:10.1142/s1793292011002342

83. S Bae, H Kim, Y Lee, X Xu, JS Park, Y Zheng, J Balakrishnan, T Lei, HR Kim, YI Song, YJ Kim, KS Kim, B Ozyilmaz, JH Ahn, BH Hong, S lijima, Roll-to-roll production of 30-inch graphene films for transparent electrodes. Nat. Nanotechnol. 5, 574-578 (2010). doi:10.1038/nnano.2010.132

84. L Gao, W Ren, H Xu, L Jin, Z Wang, T Ma, LP Ma, Z Zhang, Q Fu, LM Peng, X Bao, HM Cheng, Repeated growth and bubbling transfer of graphene with millimetre-size single-crystal grains using platinum. Nat. Commun. 3, 699 (2012). doi:10.1038/ncomms1702

85. DY Wang, IS Huang, PH Ho, SS Li, YC Yeh, DW Wang, WL Chen, YY Lee, YM Chang, CC Chen, CT Liang, CW Chen, Clean-lifting transfer of large-area residual-free graphene films. Adv. Mater. 25, $4521-4526$ (2013). doi:10.1002/ adma.201301152

86. CR Dean, AF Young, I Meric, C Lee, L Wang, S Sorgenfrei, K Watanabe, T Taniguchi, P Kim, KL Shepard, J Hone, Boron nitride substrates for highquality graphene electronics. Nat. Nanotechnol. 5, 722-726 (2010). doi:10.1038/nnano.2010.172

87. L Wang, I Meric, PY Huang, Q Gao, Y Gao, H Tran, T Taniguchi, K Watanabe, LM Campos, DA Muller, J Guo, P Kim, J Hone, KL Shepard, CR Dean, One-dimensional electrical contact to a two-dimensional material. Science 342, 614-617 (2013). doi:10.1126/science.1244358

88. L Wang, Z Chen, CR Dean, T Taniguchi, K Watanabe, LE Brus, J Hone, Negligible environmental sensitivity of graphene in a hexagonal Boron Nitride/Graphene/h-BN Sandwich structure. Acs Nano. 6, 9314-9319 (2012). doi:10.1021/nn304004s

89. T Taychatanapat, K Watanabe, T Taniguchi, P Jarillo-Herrero, Electrically tunable transverse magnetic focusing in graphene. Nat. Phys. 9, 225-229 (2013). doi:10.1038/nphys2549 
90. B Hunt, JD Sanchez-Yamagishi, AF Young, M Yankowitz, BJ LeRoy, K Watanabe, T Taniguchi, P Moon, M Koshino, P Jarillo-Herrero, RC Ashoori, Massive Dirac fermions and Hofstadter butterfly in a van der Waals Heterostructure. Science 340, 1427-1430 (2013). doi:10.1126/science.1237240

91. PS Rao, DN Sathyanarayana, Synthesis of electrically conducting copolymers of o-/m-toluidines and o-/m-amino benzoic acid in an organic peroxide system and their characterization. Synth. Met. 138, 519-527 (2003). doi:10.1016/s0379-6779(02)01253-5

92. XS Wang, HP Tang, XD Li, X Hua, Investigations on the mechanical properties of conducting polymer coating-substrate structures and their influencing factors. Int. J. Mol. Sci. 10, 5257-5284 (2009). doi:10.3390/ ijms 10125257

93. YL Wu, YN Li, BS Ong, Printed silver ohmic contacts for high-mobility organic thin-film transistors. J. Am. Chem. Soc. 128, 4202-4203 (2006). doi:10.1021/ja058725W

94. E Fortunato, A Pimentel, A Gonçalves, A Marques, R Martins, High mobility amorphous/nanocrystalline indium zinc oxide deposited at room temperature. Thin Solid Films 502, 104-107 (2006). doi:10.1016/j.tsf.2005.07.311

95. B Yaglioglu, H-Y Yeom, DC Paine, Crystallization of amorphous In[sub 2]O [sub 3]-10 wt \% ZnO thin films annealed in air. Appl. Phys. Lett. 86, 261908 (2005). doi:10.1063/1.1977209

96. T Minami, T Yamamoto, Y Toda, T Miyata, Transparent conducting zinc-codoped ITO films prepared by magnetron sputtering. Thin Solid Films 373, 189-194 (2000). doi:10.1016/s0040-6090(00)01132-9

97. F Yao, SC Lim, WJ Yu, IH Lee, F Guenes, HR Hwang, SB Yang, KP So, GH Han, AC Lee $\mathrm{YH}$, Response to gas exposure in vertically aligned multiwalled carbon nanotube electrode. J. Phys. Chem. C 114, 3659-3663 (2010). doi:10.1021/jp910678m

98. A Ghosh, YH Lee, Carbon-based electrochemical capacitors. Chem Sus Chem 5, 480-499 (2012). doi:10.1002/cssc.201100645

99. HJ Jeong, HK Choi, GY Kim, YI Song, Y Tong, SC Lim, YH Lee, Fabrication of efficient field emitters with thin multiwalled carbon nanotubes using spray method. Carbon 44, 2689-2693 (2006). doi:10.1016/j.carbon.2006.04.009

100. L Xiao, Z Chen, C Feng, L Liu, Z-Q Bai, Y Wang, L Qian, Y Zhang, Q Li, K Jiang, S Fan, Flexible, stretchable, transparent carbon nanotube thin film loudspeakers. Nano Lett. 8, 4539-4545 (2008). doi:10.1021/nl802750z

101. D Zhang, K Ryu, X Liu, E Polikarpov, J Ly, ME Tompson, C Zhou, Transparent, conductive, and flexible carbon nanotube films and their application in organic light-emitting diodes. Nano Lett. 6, 1880-1886 (2006). doi:10.1021/ nl0608543

102. J Chen, Y Liu, Al Minett, C Lynam, J Wang, GG Wallace, Flexible, aligned carbon nanotube/conducting polymer electrodes for a lithium-ion battery. Chem. Mater. 19, 3595-3597 (2007). doi:10.1021/cm070991g

103. C Feng, K Liu, J-S Wu, L Liu, J-S Cheng, Y Zhang, Y Sun, Q Li, S Fan, K Jiang Flexible, Stretchable, Transparent conducting films made from superaligned carbon nanotubes. Adv. Funct. Mater. 20, 885-891 (2010). doi:10.1002/ adfm.200901960

104. J-Y Lee, ST Connor, Y Cui, P Peumans, Solution-processed metal nanowire mesh transparent electrodes. Nano Lett. 8, 689-692 (2008). doi:10.1021/ nl073296g

105. H-Z Geng, DS Lee, KK Kim, GH Han, HK Park, YH Lee, Absorption spectroscopy of surfactant-dispersed carbon nanotube film: modulation of electronic structures. Chem. Phys. Lett. 455, 275-278 (2008). doi:10.1016/ j.cplett.2008.02.102

106. H-Z Geng, KK Kim, K Lee, GY Kim, HK Choi, DS Lee, KH An, YH Lee, Y Chang, YS Lee, B Kim, YJ Lee, Dependence of material quality on performance of flexible transparent conducting films with single-walled carbon nanotubes. Nano 2, 157-167 (2007). doi:10.1142/s1793292007000532

107. M Razeghi, H-Z Geng, KK Kim, YH Lee, D Pribat, YH Lee, Recent progress in carbon nanotube-based flexible transparent conducting film. Proc. of SPIE. 7037, 70370A-1-70370A-14 (2008). doi:10.1117/12.796143

108. KK Kim, S-M Yoon, HK Park, H-J Shin, SM Kim, JJ Bae, Y Cui, JM Kim, J-Y Choi, YH Lee, Doping strategy of carbon nanotubes with redox chemistry. New J. Chem. 34, 2183-2188 (2010). doi:10.1039/c0nj00138d

109. SM Kim, KK Kim, YW Jo, MH Park, SJ Chae, DL Duong, CW Yang, J Kong, YH Lee, Role of anions in the AuCl3-Doping of carbon nanotubes. Acs Nano. 5, 1236-1242 (2011). doi:10.1021/nn1028532

110. KK Kim, JJ Bae, HK Park, SM Kim, H-Z Geng, KA Park, H-J Shin, S-M Yoon, A Benayad, J-Y Choi, YH Lee, Fermi level engineering of single-walled carbon nanotubes by AuCl3 doping. J. Am. Chem. Soc. 130, 12757-12761 (2008). doi:10.1021/ja8038689
111. F Yao, F Gunes, HQ Ta, SM Lee, SJ Chae, KY Sheem, CS Cojocaru, SS Xie, YH Lee, Diffusion mechanism of lithium ion through basal plane of layered graphene. J. Am. Chem. Soc. 134, 8646-8654 (2012). doi:10.1021/ja301586m

112. B Kumar, KY Lee, H-K Park, SJ Chae, YH Lee, S-W Kim, Controlled growth of semiconducting nanowire, nanowall, and hybrid nanostructures on graphene for piezoelectric nanogenerators. Acs Nano. 5, 4197-4204 (2011) doi:10.1021/nn200942s

113. D Choi, MY Choi, WM Choi, HJ Shin, HK Park, JS Seo, J Park, SM Yoon, SJ Chae, YH Lee, SW Kim, JY Choi, SY Lee, JM Kim, Fully rollable transparent nanogenerators based on graphene electrodes. Adv. Mater. 22, 2187-2192 (2010). doi:10.1002/adma.200903815

114. KK Kim, A Reina, Y Shi, H Park, LJ Li, YH Lee, J Kong, Enhancing the conductivity of transparent graphene films via doping. Nanotechnology 21, 285205 (2010). doi:10.1088/0957-4484/21/28/285205

115. H-J Shin, WM Choi, D Choi, GH Han, S-M Yoon, H-K Park, S-W Kim, YW Jin, SY Lee, JM Kim, J-Y Choi, YH Lee, Control of electronic structure of graphene by various dopants and their effects on a nanogenerator. J. Am. Chem. Soc. 132, 15603-15609 (2010). doi:10.1021/ja105140e

116. F Guenes, GH Han, KK Kim, ES Kim, SJ Chae, MH Park, H-K Jeong, SC Lim, YH Lee, Large-area graphene-based flexible transparent conducting films. Nano 4, 83-90 (2009). doi:10.1142/s1793292009001538

117. F Gunes, H-J Shin, C Biswas, GH Han, ES Kim, SJ Chae, J-Y Choi, YH Lee, Layer-by-layer doping of few-layer graphene film. Acs Nano. 4, 4595-4600 (2010). doi:10.1021/nn1008808

118. WH Lee, J Park, SH Sim, SB Jo, KS Kim, BH Hong, K Cho, Transparent flexible organic transistors based on monolayer graphene electrodes on plastic. Adv. Mater. 23, 1752-1756 (2011). doi:10.1002/adma.201004099

119. Y Chen, Y Xu, K Zhao, X Wan, J Deng, W Yan, Towards flexible all-carbon electronics: flexible organic field-effect transistors and inverter circuits using solution-processed all-graphene source/drain/gate electrodes. Nano Res. 3, 714-721 (2010). doi:10.1007/s12274-010-0035-3

120. C Li, Z Li, H Zhu, K Wang, J Wei, X Li, P Sun, H Zhang, D Wu, Graphene Nano-"patches" on a carbon nanotube network for highly transparent/ conductive thin film applications. J. Phys. Chem. C 114, 14008-14012 (2010). doi:10.1021/jp1041487

121. T-K Hong, DW Lee, HJ Choi, HS Shin, B-S Kim, Transparent, flexible conducting hybrid multi layer thin films of multiwalled carbon nanotubes with graphene nanosheets. Acs Nano. 4, 3861-3868 (2010). doi:10.1021/ $\mathrm{nn} 100897 \mathrm{~g}$

122. VC Tung, L-M Chen, MJ Allen, JK Wassei, K Nelson, RB Kaner, Y Yang, Low-temperature solution processing of graphene-carbon nanotube hybrid materials for high-performance transparent conductors. Nano Lett. 9. 1949-1955 (2009). doi:10.1021/nl9001525

123. WJ Yu, SY Lee, SH Chae, D Perello, GH Han, M Yun, YH Lee, Small hysteresis nanocarbon-based integrated circuits on flexible and transparent plastic substrate. Nano letters. 11, 1344-1350 (2011). doi:10.1021/nl104488z

124. Y Zhu, Z Sun, Z Yan, Z Jin, JM Tour, Rational design of hybrid graphene films for high-performance transparent electrodes. Acs Nano. 5, 6472-6479 (2011). doi:10.1021/nn201696g

125. Q Cao, SJ Han, GS Tulevski, Y Zhu, DD Lu, W Haensch, Arrays of singlewalled carbon nanotubes with full surface coverage for high-performance electronics. Nat. Nanotechnol. 8, 180-186 (2013). doi:10.1038/ nnano. 2012.257

126. VK Sangwan, RP Ortiz, JMP Alaboson, JD Emery, MJ Bedzyk, LJ Lauhon, TJ Marks, MC Hersam, Fundamental performance limits of carbon nanotube thin-film transistors achieved using hybrid molecular dielectrics. Acs Nano. 6, 7480-7488 (2012). doi:10.1021/nn302768h

127. I Meric, MY Han, AF Young, B Ozyilmaz, P Kim, KL Shepard, Current saturation in zero-bandgap, top-gated graphene field-effect transistors. Nat. Nanotechnol. 3, 654-659 (2008). doi:10.1038/nnano.2008.268

128. C Biswas, SY Lee, L Thuc Hue, A Ghosh, D Quoc Nguyen, YH Lee, Chemically doped random network carbon nanotube $\mathrm{p}-n$ junction diode for rectifier. Acs Nano. 5, 9817-9823 (2011). doi:10.1021/nn203391h

129. A Javey, H Kim, M Brink, Q Wang, A Ural, J Guo, P Mclntyre, P McEuen, M Lundstrom, H Dai, High-k dielectrics for advanced carbon-nanotube transistors and logic gates. Nat. Mater. 1, 241-246 (2002). doi:10.1038/ nmat769

130. A Javey, J Guo, DB Farmer, Q Wang, E Yenilmez, RG Gordon, M Lundstrom, HJ Dai, Self-aligned ballistic molecular transistors and electrically parallel nanotube arrays. Nano Lett. 4, 1319-1322 (2004). doi:10.1021/nl049222b 
131. C Klinke, J Chen, A Afzali, P Avouris, Charge transfer induced polarity switching in carbon nanotube transistors. Nano Lett. 5, 555-558 (2005). doi:10.1021/nl048055c

132. SJ Tans, ARM Verschueren, C Dekker, Room-temperature transistor based on a single carbon nanotube. Nature 393, 49-52 (1998)

133. A Javey, J Guo, Q Wang, M Lundstrom, HJ Dai, Ballistic carbon nanotube field-effect transistors. Nature 424, 654-657 (2003). doi:10.1038/ nature01797

134. A Bachtold, P Hadley, T Nakanishi, C Dekker, Logic circuits with carbon nanotube transistors. Science 294, 1317-1320 (2001). doi:10.1126/ science.1065824

135. DJ Perello, SC Lim, SJ Chae, I Lee, MJ Kim, YH Lee, M Yun, Thermionic field emission transport in carbon nanotube transistors. Acs Nano. 5, 1756-1760 (2011). doi:10.1021/nn102343k

136. C Kocabas, SJ Kang, T Ozel, M Shim, JA Rogers, Improved synthesis of aligned arrays of single-walled carbon nanotubes and their implementation in thin film type transistors. J. Phys. Chem. C 111, 17879-17886 (2007). doi:10.1021/jp071387w

137. L Ding, D Yuan, J Liu, Growth of high-density parallel arrays of long single-walled carbon nanotubes on quartz substrates. J. Am. Chem. Soc. 130, 5428-+ (2008). doi:10.1021/ja8006947

138. WJ Yu, UJ Kim, BR Kang, IH Lee, E-H Lee, YH Lee, Adaptive logic circuits with doping-free ambipolar carbon nanotube transistors. Nano Lett. 9, 1401-1405 (2009). doi:10.1021/nl803066v

139. SW Lee, SY Lee, SC Lim, K Y-d, J-S Yoon, K Uh, YH Lee, Positive gate bias stress instability of carbon nanotube thin film transistors. Appl. Phys. Lett. 101, (2012). doi:10.1063/1.4740084

140. BJ Kim, H Jang, SK Lee, BH Hong, JH Ahn, JH Cho, High-performance flexible graphene field effect transistors with ion gel gate dielectrics. Nano letters. 10, 3464-3466 (2010). doi:10.1021/nl101559n

141. AK Geim, KS Novoselov, The rise of graphene. Nat. Mater. 6, 183-191 (2007). doi:10.1038/nmat1849

142. J Lee, TJ Ha, KN Parrish, SF Chowdhury, L Tao, A Dodabalapur, D Akinwande, High-performance current saturating graphene field-effect transistor with hexagonal boron nitride dielectric on flexible polymeric substrates. IEEE Electron. Dev. L. 34, 172-174 (2013). doi:10.1109/ led.2012.2233707

143. C Yan, JH Cho, JH Ahn, Graphene-based flexible and stretchable thin film transistors. Nanoscale. 4, 4870-4882 (2012). doi:10.1039/c2nr30994g

144. F Schwierz, ELECTRONICS Industry-compatible graphene transistors. Nature 472, 41-42 (2011)

145. L Chico, VH Crespi, LX Benedict, SG Louie, ML Cohen, Pure carbon nanoscale devices: nanotube heterojunctions. Phys. Rev. Lett. 76, 971-974 (1996). doi:10.1103/PhysRevLett.76.971

146. HJ Dai, EW Wong, CM Lieber, Probing electrical transport in nanomaterials: conductivity of individual carbon nanotubes. Science 272, 523-526 (1996). doi:10.1126/science. 272.5261 .523

147. PG Collins, MS Arnold, P Avouris, Engineering carbon nanotubes and nanotube circuits using electrical breakdown. Science 292, 706-709 (2001). doi:10.1126/science.1058782

148. P Avouris, Carbon nanotube electronics. Chem. Phys. 281, 429-445 (2002). doi:10.1016/s0301-0104(02)00376-2

149. Q Cao, HS Kim, N Pimparkar, JP Kulkarni, C Wang, M Shim, K Roy, MA Alam, JA Rogers, Medium-scale carbon nanotube thin-film integrated circuits on flexible plastic substrates. Nature 454, 495-500 (2008). doi:10.1038/ nature07110

150. AA Green, MC Hersam, Processing and properties of highly enriched double-wall carbon nanotubes. Nat. Nanotechnol. 4, 64-70 (2009). doi:10.1038/nnano.2008.364

151. S Ghosh, SM Bachilo, RB Weisman, Advanced sorting of single-walled carbon nanotubes by nonlinear density-gradient ultracentrifugation. Nat. Nanotechnol. 5, 443-450 (2010). doi:10.1038/nnano.2010.68

152. H Liu, D Nishide, T Tanaka, H Kataura, Large-scale single-chirality separation of single-wall carbon nanotubes by simple gel chromatography. Nat. Commun. 2, 309 (2011). doi:10.1038/ncomms1313

153. MS Arnold, AA Green, JF Hulvat, SI Stupp, MC Hersam, Sorting carbon nanotubes by electronic structure using density differentiation. Nat. Nanotechnol. 1, 60-65 (2006). doi:10.1038/nnano.2006.52

154. JB Oostinga, HB Heersche, X Liu, AF Morpurgo, LM Vandersypen, Gate-induced insulating state in bilayer graphene devices. Nat. Mater. 7, 151-157 (2008). doi:10.1038/nmat2082
155. T Ohta, A Bostwick, T Seyller, K Horn, E Rotenberg, Controlling the electronic structure of bilayer graphene. Science 313, 951-954 (2006). doi:10.1126/ science.1130681

156. J Velasco Jr, L Jing, W Bao, Y Lee, P Kratz, V Aji, M Bockrath, CN Lau, C Varma, R Stillwell, D Smirnov, F Zhang, J Jung, AH MacDonald, Transport spectroscopy of symmetry-broken insulating states in bilayer graphene. Nat. Nanotechnol. 7, 156-160 (2012). doi:10.1038/nnano.2011.251

157. Y Zhang, TT Tang, C Girit, Z Hao, MC Martin, A Zettl, MF Crommie, YR Shen, F Wang, Direct observation of a widely tunable bandgap in bilayer graphene. Nature 459, 820-823 (2009). doi:10.1038/nature08105

158. F Xia, DB Farmer, YM Lin, P Avouris, Graphene field-effect transistors with high on/off current ratio and large transport band gap at room temperature. Nano Lett. 10, 715-718 (2010). doi:10.1021/n19039636

159. X Li, KM Borysenko, MB Nardelli, KW Kim, Electron transport properties of bilayer graphene. Phys. Rev. B 84, (2011). doi:10.1103/PhysRevB.84.195453

160. J Park, SB Jo, YJ Yu, Y Kim, JW Yang, WH Lee, HH Kim, BH Hong, P Kim, K Cho, KS Kim, Single-Gate Bandgap Opening of Bilayer Graphene by Dual Molecular Doping. Advanced materials. (2011). doi:10.1002/ adma.201103411

161. DL Duong, SM Lee, SH Chae, QH Ta, SY Lee, GH Han, JJ Bae, YH Lee, Band-gap engineering in chemically conjugated bilayer graphene: Ab initio calculations. Phys. Rev. B 85, (2012). doi:10.1103/PhysRevB.85.205413

162. L Jiao, L Zhang, X Wang, G Diankov, H Dai, Narrow graphene nanoribbons from carbon nanotubes. Nature 458, 877-880 (2009). doi:10.1038/ nature07919

163. X Li, X Wang, L Zhang, S Lee, H Dai, Chemically derived, ultrasmooth graphene nanoribbon semiconductors. Science 319, 1229-1232 (2008). doi:10.1126/science.1150878

164. M Han, B Özyilmaz, Y Zhang, P Kim, Energy band-gap engineering of graphene nanoribbons. Phys. Rev. Lett. 98, (2007). doi:10.1103/ PhysRevLett.98.206805

165. X Wang, Y Ouyang, X Li, H Wang, J Guo, H Dai, Room-temperature allsemiconducting Sub-10-nm graphene nanoribbon field-effect transistors. Phys. Rev. Lett. 100, (2008). doi:10.1103/PhysRevLett.100.206803

166. J Bai, X Zhong, S Jiang, Y Huang, X Duan, Graphene nanomesh. Nat. Nanotechnol. 5, 190-194 (2010). doi:10.1038/nnano.2010.8

167. M Kim, NS Safron, E Han, MS Arnold, P Gopalan, Fabrication and characterization of large-area, semiconducting nanoperforated graphene materials. Nano letters. 10, 1125-1131 (2010). doi:10.1021/ nl9032318

168. Y-B Tang, L-C Yin, Y Yang, X-H Bo, Y-L Cao, H-E Wang, W-J Zhang, I Bello, S-T Lee, H-M Cheng, C-S Lee, Tunable band gaps and p-Type transport properties of boron-doped graphenes by controllable ion doping using reactive microwave plasma. Acs Nano. 6, 1970-1978 (2012). doi:10.1021/ nn3005262

169. D Wei, Y Liu, Y Wang, H Zhang, L Huang, G Yu, Synthesis of N-Doped graphene by chemical vapor deposition and its electrical properties. Nano Lett. 9, 1752-1758 (2009). doi:10.1021/nl803279t

170. R Balog, B Jorgensen, L Nilsson, M Andersen, E Rienks, M Bianchi, M Fanetti, E Laegsgaard, A Baraldi, S Lizzit, Z Sljivancanin, F Besenbacher, B Hammer, TG Pedersen, $\mathrm{P}$ Hofmann, L Hornekaer, Bandgap opening in graphene induced by patterned hydrogen adsorption. Nat. Mater. 9, 315-319 (2010). doi:10.1038/nmat2710

171. J Chen, C Klinke, A Afzali, P Avouris, Self-aligned carbon nanotube transistors with charge transfer doping. Appl. Phys. Lett. 86, 123108 (2005). doi:10.1063/1.1888054

172. MS Strano, CA Dyke, ML Usrey, PW Barone, MJ Allen, H Shan, C Kittrell, RH Hauge, JM Tour, RE Smalley, Electronic structure control of single-walled carbon nanotube functionalization. Science 301, 1519-1522 (2003). doi:10.1126/science.1087691

173. DL Duong, IH Lee, KK Kim, J Kong, SM Lee, YH Lee, Carbon nanotube doping mechanism in a salt solution and hygroscopic effect: density functional theory. Acs Nano. 4, 5430-5436 (2010). doi:10.1021/ nn1011489

174. S-M Yoon, UJ Kim, A Benayad, IH Lee, H Son, H-J Shin, WM Choi, YH Lee, YW Jin, E-H Lee, SY Lee, J-Y Choi, JM Kim, Thermal conversion of electronic and electrical properties of AuCl3-Doped single-walled carbon nanotubes. Acs Nano. 5, 1353-1359 (2011). doi:10.1021/nn103055u

175. S Heinze, J Tersoff, R Martel, V Derycke, J Appenzeller, P Avouris, Carbon nanotubes as schottky barrier transistors. Phys. Rev. Lett. 89, (2002). doi:10.1103/PhysRevLett.89.106801 
176. T Takenobu, T Kanbara, N Akima, T Takahashi, M Shiraishi, K Tsukagoshi, H Kataura, Y Aoyagi, Y Iwasa, Control of carrier density by a solution method in carbon-nanotube devices. Adv. Mater. 17, 2430-2434 (2005). doi:10.1002/ adma.200500759

177. V Derycke, R Martel, J Appenzeller, P Avouris, Controlling doping and carrier injection in carbon nanotube transistors. Appl. Phys. Lett. 80, 2773 (2002). doi:10.1063/1.1467702

178. C Zhou, Modulated chemical doping of individual carbon nanotubes. Science 290, 1552-1555 (2000). doi:10.1126/science.290.5496.1552

179. IH Lee, UJ Kim, H Bin Son, S-M Yoon, F Yao, WJ Yu, DL Duong, J-Y Choi, JM Kim, EH Lee, YH Lee, Hygroscopic effects on AuCl3-Doped carbon nanotubes. J. Phys. Chem. C 114, 11618-11622 (2010). doi:10.1021/ .jp1036662

180. SM Kim, JH Jang, KK Kim, HK Park, JJ Bae, WJ Yu, IH Lee, G Kim, DD Loc, UJ Kim, E-H Lee, H-J Shin, J-Y Choi, YH Lee, Reduction-controlled viologen in bisolvent as an environmentally stable n-Type dopant for carbon nanotubes. J. Am. Chem. Soc. 131, 327-331 (2009). doi:10.1021/ja807480g

181. SY Lee, SW Lee, SM Kim, WJ Yu, YW Jo, YH Lee, Scalable complementary logic gates with chemically doped semiconducting carbon nanotube transistors. Acs Nano 5, 2369-2375 (2011). doi:10.1021/nn200270e

182. BR Kang, WJ Yu, KK Kim, HK Park, SM Kim, Y Park, G Kim, H-J Shin, UJ Kim, E-H Lee, J-Y Choi, YH Lee, Restorable type conversion of carbon nanotube transistor using pyrolytically controlled antioxidizing photosynthesis coenzyme. Adv. Funct. Mater. 19, 2553-2559 (2009). doi:10.1002/ adfm.200801712

183. SM Kim, YW Jo, KK Kim, DL Duong, H-J Shin, JH Han, J-Y Choi, J Kong, YH Lee, Transparent organic P-Dopant in carbon nanotubes: Bis(trifluoromethanesulfonyl)imide. Acs Nano. 4, 6998-7004 (2010). doi:10.1021/nn102175h

184. HK Jeong, K-j Kim, SM Kim, YH Lee, Modification of the electronic structures of graphene by viologen. Chem. Phys. Lett. 498, 168-171 (2010). doi:10.1016/j.cplett.2010.08.065

185. A Benayad, H-J Shin, HK Park, S-M Yoon, KK Kim, MH Jin, H-K Jeong, JC Lee, J-Y Choi, YH Lee, Controlling work function of reduced graphite oxide with Au-ion concentration. Chem. Phys. Lett. 475, 91-95 (2009). doi:10.1016/ j.cplett.2009.05.023

186. WJ Yu, L Liao, SH Chae, YH Lee, X Duan, Toward tunable band gap and tunable dirac point in bilayer graphene with molecular doping. Nano letters. 11, 4759-4763 (2011). doi:10.1021/nl2025739

187. V Derycke, R Martel, J Appenzeller, P Avouris, Carbon nanotube inter- and intramolecular logic gates. Nano Lett. 1, 453-456 (2001). doi:10.1021/ nl015606f

188. GU Sumanasekera, CKW Adu, S Fang, PC Eklund, Effects of gas adsorption and collisions on electrical transport in single-walled carbon nanotubes. Phys. Rev. Lett. 85, 1096-1099 (2000). doi:10.1103/PhysRevLett.85.1096

189. PG Collins, Extreme oxygen sensitivity of electronic properties of carbon nanotubes. Science 287, 1801-1804 (2000). doi:10.1126/ science.287.5459.1801

190. WJ Yu, BR Kang, IH Lee, YS Min, YH Lee, Majority carrier type conversion with floating gates in carbon nanotube transistors. Adv. Mater. 21, 4821-4824 (2009). doi:10.1002/adma.200900911

191. WJ Yu, YH Lee, Strategy for carrier control in carbon nanotube transistors. ChemSusChem 4, 890-904 (2011). doi:10.1002/cssc.201000412

192. M Bockrath, J Hone, A Zettl, PL McEuen, AG Rinzler, RE Smalley, Chemical doping of individual semiconducting carbon-nanotube ropes. Phys. Rev. B 61, 10606-10608 (2000)

193. MH Yang, KBK Teo, WI Milne, DG Hasko, Carbon nanotube Schottky diode and directionally dependent field-effect transistor using asymmetrical contacts. Appl. Phys. Lett. 87, 253116 (2005). doi:10.1063/1.2149991

194. Y Nosho, Y Ohno, S Kishimoto, T Mizutani, Relation between conduction property and work function of contact metal in carbon nanotube fieldeffect transistors. Nanotechnology 17, 3412-3415 (2006). doi:10.1088/ 0957-4484/17/14/011

195. Y Lee, S Bae, H Jang, S Jang, SE Zhu, SH Sim, YI Song, BH Hong, JH Ahn, Wafer-scale synthesis and transfer of graphene films. Nano letters. 10, 490-493 (2010). doi:10.1021/nl903272n

196. Q Cao, JA Rogers, Ultrathin films of single-walled carbon nanotubes for electronics and sensors: a review of fundamental and applied aspects. Adv. Mater. 21, 29-53 (2009). doi:10.1002/adma.200801995

197. S Jang, H Jang, Y Lee, D Suh, S Baik, BH Hong, JH Ahn, Flexible, transparent single-walled carbon nanotube transistors with graphene electrodes. Nanotechnology 21, 425201 (2010). doi:10.1088/0957-4484/21/42/425201
198. JH Chen, M Ishigami, C Jang, DR Hines, MS Fuhrer, ED Williams, Printed Graphene Circuits. Adv. Mater. 19, 3623-3627 (2007). doi:10.1002/ adma.200701059

199. JU Park, S Nam, MS Lee, CM Lieber, Synthesis of monolithic graphenegraphite integrated electronics. Nat. Mat. 11, 120-125 (2012). doi:10.1038/ nmat3169

200. DM Sun, C Liu, WC Ren, HM Cheng, A review of carbon nanotube- and graphene-based flexible thin-film transistors. Small 9, 1188-1205 (2013). doi:10.1002/smll.201203154

201. C-C Lu, Y-C Lin, C-H Yeh, J-C Huang, P-W Chiu, High mobility flexible graphene field-effect transistors with self-healing gate dielectrics. Acs Nano. 6, 4469-4474 (2012). doi:10.1021/nn301199j

202. T Kim, H Kim, SW Kwon, Y Kim, WK Park, DH Yoon, AR Jang, HS Shin, KS Suh, WS Yang, Large-scale graphene micropatterns via self-assemblymediated process for flexible device application. Nano letters. 12, 743-748 (2012). doi:10.1021/nl203691d

203. SJ Park, OS Kwon, SH Lee, HS Song, TH Park, J Jang, Ultrasensitive flexible graphene based field-effect transistor (FET)-type bioelectronic nose. Nano letters. 12, 5082-5090 (2012). doi:10.1021/nl301714x

204. Q Cao, SH Hur, ZT Zhu, YG Sun, CJ Wang, MA Meitl, M Shim, JA Rogers, Highly Bendable, transparent thin-film transistors that use carbon-nanotubebased conductors and semiconductors with elastomeric dielectrics. Adv. Mater. 18, 304-309 (2006). doi:10.1002/adma.200501740

205. DM Sun, MY Timmermans, Y Tian, AG Nasibulin, El Kauppinen, S Kishimoto, T Mizutani, Y Ohno, Flexible high-performance carbon nanotube integrated circuits. Nat. Nanotechnol. 6, 156-161 (2011). doi:10.1038/nnano.2011.1

206. BJ Kim, S-K Lee, MS Kang, J-H Ahn, JH Cho, Coplanar-gate transparent graphene transistors and inverters on plastic. Acs Nano. 6, 8646-8651 (2012). doi:10.1021/nn3020486

207. H Wang, D Nezich, J Kong, T Palacios, Graphene frequency multipliers. IEEE Electron. Dev. L. 30, 547-549 (2009). doi:10.1109/led.2009.2016443

208. X Yang, G Liu, AA Balandin, K Mohanram, Triple-mode single-transistor graphene amplifier and its applications. Acs Nano. 4, 5532-5538 (2010). doi:10.1021/nn1021583

209. Z Wang, Z Zhang, H Xu, L Ding, S Wang, L-M Peng, A high-performance top-gate graphene field-effect transistor based frequency doubler. Appl. Phys. Lett. 96, 173104 (2010). doi:10.1063/1.3413959

210. H Wang, A Hsu, J Wu, J Kong, T Palacios, RF Graphene-Based Ambipolar, Mixers. IEEE Electron. Dev. Lett. 31, 906-908 (2010). doi:10.1109/ led.2010.2052017

211. A Hsu, H Wang, KK Kim, J Kong, T Palacios, High frequency performance of graphene transistors grown by chemical vapor deposition for mixed signal applications. Jpn. J. Appl. Phys. 50, 070114 (2011). doi:10.1143/ jjap.50.070114

212. S Lee, K Lee, CH Liu, GS Kulkarni, Z Zhong, Flexible and transparent allgraphene circuits for quaternary digital modulations. Nat. Commun. 3, 1018 (2012). doi:10.1038/ncomms2021

213. T Takahashi, K Takei, AG Gillies, RS Fearing, A Javey, Carbon nanotube active-matrix backplanes for conformal electronics and sensors. Nano letters. 11, 5408-5413 (2011). doi:10.1021/nl203117h

214. JA Rogers, Z Bao, K Baldwin, A Dodabalapur, B Crone, VR Raju, V Kuck, H Katz, K Amundson, J Ewing, P Drzaic, Paper-like electronic displays: largearea rubber-stamped plastic sheets of electronics and microencapsulated electrophoretic inks. Proc. Natl. Acad. Sci. U. S. A. 98, 4835-4840 (2001). doi:10.1073/pnas.091588098

215. C Wang, D Hwang, Z Yu, K Takei, J Park, T Chen, B Ma, A Javey, Userinteractive electronic skin for instantaneous pressure visualization. Nat Mater. 12, 899-904 (2013). doi:10.1038/nmat3711

216. Z-g Wang, Y-f Chen, P-j Li, X Hao, J-b Liu, R Huang, Y-r Li, Flexible Graphene-Based Electroluminescent Devices. Acs Nano. 5, 7149-7154 (2011). doi:10.1021/nn2018649

217. C Sire, F Ardiaca, S Lepilliet, JW Seo, MC Hersam, G Dambrine, H Happy, $\checkmark$ Derycke, Flexible gigahertz transistors derived from solution-based single-layer graphene. Nano letters. 12, 1184-1188 (2012). doi:10.1021/ nl203316r

218. OM Nayfeh, Graphene transistors on mechanically flexible polyimide incorporating atomic-layer-deposited gate dielectric. IEEE Electron. Dev. L. 32, 1349-1351 (2011). doi:10.1109/led.2011.2163489

219. J Lee, T-J Ha, H Li, KN Parrish, M Holt, A Dodabalapur, RS Ruoff, D Akinwande, $25 \mathrm{GHz}$ embedded-gate graphene transistors with high-K dielectrics on extremely flexible plastic sheets. Acs Nano. 7, 7744-7750 (2013) 
220. N Petrone, I Meric, J Hone, KL Shepard, Graphene field-effect transistors with gigahertz-frequency power gain on flexible substrates. Nano letters. 13, 121-125 (2013). doi:10.1021/nl303666m

221. L Liao, YC Lin, M Bao, R Cheng, J Bai, Y Liu, Y Qu, KL Wang, Y Huang, X Duan, High-speed graphene transistors with a self-aligned nanowire gate. Nature 467, 305-308 (2010). doi:10.1038/nature09405

222. C Heo, SY Lee, A Jo, S Jung, M Suh, YH Lee, Flexible, transparent, and noncytotoxic graphene electric field stimulator for effective cerebral blood volume enhancement. Acs Nano. 7, 4869-4878 (2013). doi:10.1021/ nn305884w

223. WJ Yu, SH Chae, SY Lee, DL Duong, YH Lee, Ultra-transparent, flexible single-walled carbon nanotube non-volatile memory device with an oxygen-decorated graphene electrode. Adv. Mater. 23, 1889-1893 (2011). doi:10.1002/adma.201004444

224. B Park, K Cho, S Kim, S Kim, Transparent nano-floating gate memory on glass. Nanotechnology 21, 335201 (2010). doi:10.1088/0957-4484/21/33/ 335201

225. Y Ji, S Lee, B Cho, S Song, T Lee, Flexible organic memory devices with multilayer graphene electrodes. Acs Nano. 5, 5995-6000 (2011). doi:10.1021/ $\mathrm{nn} 201770 \mathrm{~s}$

226. HY Jeong, JY Kim, JW Kim, JO Hwang, JE Kim, JY Lee, TH Yoon, BJ Cho, SO Kim, RS Ruoff, SY Choi, Graphene oxide thin films for flexible nonvolatile memory applications. Nano letters. 10, 4381-4386 (2010). doi:10.1021/ nl101902k

227. TS Hansen, K West, O Hassager, NB Larsen, Highly stretchable and conductive polymer material made from Poly(3,4-ethylenedioxythiophene) and polyurethane elastomers. Adv. Funct. Mater. 17, 3069-3073 (2007). doi:10.1002/adfm.200601243

228. F Xu, W Lu, Y Zhu, Controlled 3D buckling of silicon nanowires for stretchable electronics. Acs Nano. 5, 672-678 (2011). doi:10.1021/nn103189z

229. P Lee, J Lee, H Lee, J Yeo, S Hong, KH Nam, D Lee, SS Lee, SH Ko, Highly stretchable and highly conductive metal electrode by very long metal nanowire percolation network. Adv. Mater. 24, 3326-3332 (2012). doi:10.1002/adma.201200359

230. C Yu, C Masarapu, J Rong, B Wei, H Jiang, Stretchable supercapacitors based on buckled single-walled carbon-nanotube macrofilms. Adv. Mater. 21, 4793-4797 (2009). doi:10.1002/adma.200901775

231. F Xu, X Wang, Y Zhu, Y Zhu, Wavy ribbons of carbon nanotubes for stretchable conductors. Adv. Funct. Mater. 22, 1279-1283 (2012). doi:10.1002/adfm.201102032

232. J Xiao, A Carlson, ZJ Liu, Y Huang, H Jiang, JA Rogers, Stretchable and compressible thin films of stiff materials on compliant wavy substrates. Appl. Phys. Lett. 93, 013109 (2008). doi:10.1063/1.2955829

233. J Jeong, S Kim, J Cho, Y Hong, Stable stretchable silver electrode directly deposited on wavy elastomeric substrate. IEEE Electron. Dev. Lett. 30, 1284-1286 (2009). doi:10.1109/led.2009.2033723

234. Y Sun, V Kumar, I Adesida, JA Rogers, Buckled and wavy ribbons of gaas for high-performance electronics on elastomeric substrates. Adv. Mater. 18, 2857-2862 (2006). doi:10.1002/adma.200600646

235. DY Khang, HQ Jiang, Y Huang, JA Rogers, A stretchable form of singlecrystal silicon for high-performance electronics on rubber substrates. Science 311, 208-212 (2006). doi:10.1126/science.1121401

236. C Hierold, A Jungen, C Stampfer, T Helbling, Nano electromechanical sensors based on carbon nanotubes. Sensors Actuators A Phys. 136, 51-61 (2007). doi:10.1016/j.sna.2007.02.007

237. TW Tombler, CW Zhou, L Alexseyev, J Kong, HJ Dai, L Lei, CS Jayanthi, MJ Tang, SY Wu, Reversible electromechanical characteristics of carbon nanotubes under local-probe manipulation. Nature 405, 769-772 (2000)

238. H Maune, M Bockrath, Elastomeric carbon nanotube circuits for local strain sensing. Appl. Phys. Lett. 89, 173131 (2006). doi:10.1063/1.2358821

239. DJ Lipomi, M Vosgueritchian, BC Tee, SL Hellstrom, JA Lee, CH Fox, Z Bao, Skin-like pressure and strain sensors based on transparent elastic films of carbon nanotubes. Nat. Nanotechnol. 6, 788-792 (2011). doi:10.1038/ nnano.2011.184

240. KS Kim, Y Zhao, H Jang, SY Lee, JM Kim, KS Kim, JH Ahn, P Kim, JY Choi, $\mathrm{BH}$ Hong, Large-scale pattern growth of graphene films for stretchable transparent electrodes. Nature 457, 706-710 (2009). doi:10.1038/ nature07719

241. Z Chen, W Ren, L Gao, B Liu, S Pei, HM Cheng, Three-dimensional flexible and conductive interconnected graphene networks grown by chemical vapour deposition. Nat. Mater. 10, 424-428 (2011). doi:10.1038/nmat3001
242. ME Roberts, N Queralto, SCB Mannsfeld, BN Reinecke, W Knoll, Z Bao, Cross-linked polymer gate dielectric films for low-voltage organic transistors. Chem. Mater. 21, 2292-2299 (2009). doi:10.1021/cm900637p

243. DM Sun, MY Timmermans, A Kaskela, AG Nasibulin, S Kishimoto, T Mizutani, El Kauppinen, Y Ohno, Mouldable all-carbon integrated circuits. Nat. Commun. 4, 2302 (2013). doi:10.1038/ncomms3302

244. SK Lee, BJ Kim, H Jang, SC Yoon, C Lee, BH Hong, JA Rogers, JH Cho, JH Ahn, Stretchable graphene transistors with printed dielectrics and gate electrodes. Nano letters. 11, 4642-4646 (2011). doi:10.1021/nl202134z

245. SH Chae, WJ Yu, JJ Bae, DL Duong, D Perello, HY Jeong, QH Ta, TH Ly, QA Vu, M Yun, X Duan, YH Lee, Transferred wrinkled Al2O3 for highly stretchable and transparent graphene-carbon nanotube transistors. Nat. Mater. 12, 403-409 (2013). doi:10.1038/nmat3572

246. T Yamada, Y Hayamizu, Y Yamamoto, Y Yomogida, A Izadi-Najafabadi, DN Futaba, K Hata, A stretchable carbon nanotube strain sensor for humanmotion detection. Nat. Nanotechnol. 6, 296-301 (2011). doi:10.1038/ nnano.2011.36

doi:10.1186/s40580-014-0015-5

Cite this article as: Chae and Lee: Carbon nanotubes and graphene towards soft electronics. Nano Convergence 2014 1:15.

\section{Submit your manuscript to a SpringerOpen ${ }^{\odot}$ journal and benefit from:}

- Convenient online submission

- Rigorous peer review

- Immediate publication on acceptance

- Open access: articles freely available online

- High visibility within the field

- Retaining the copyright to your article

Submit your next manuscript at $>$ springeropen.com 